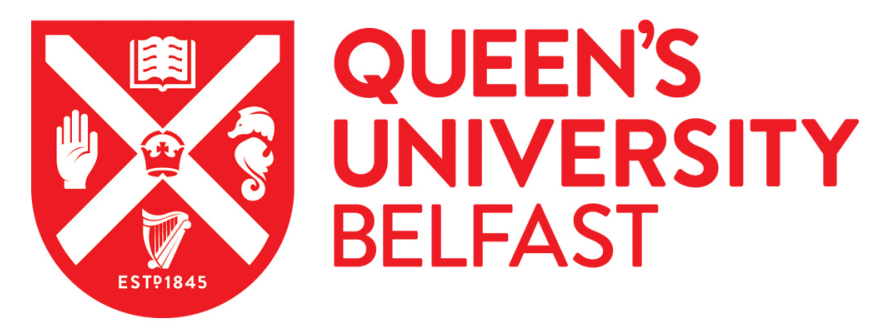

\title{
Length- And Thickness-Dependent Optical Response of Liquid- Exfoliated Transition Metal Dichalcogenides
}

Synnatschke, K., Cieslik, P. A., Harvey, A., Castellanos-Gomez, A., Tian, T., Shih, C. J., Chernikov, A., Santos, E. J. G., Coleman, J. N., \& Backes, C. (2019). Length- And Thickness-Dependent Optical Response of LiquidExfoliated Transition Metal Dichalcogenides. Chemistry of Materials.

https://doi.org/10.1021/acs.chemmater.9b02905

Published in:

Chemistry of Materials

Document Version:

Peer reviewed version

Queen's University Belfast - Research Portal:

Link to publication record in Queen's University Belfast Research Portal

Publisher rights

Copyright 2019 ACS. This work is made available online in accordance with the publisher's policies. Please refer to any applicable terms of use of the publisher.

\section{General rights}

Copyright for the publications made accessible via the Queen's University Belfast Research Portal is retained by the author(s) and / or other copyright owners and it is a condition of accessing these publications that users recognise and abide by the legal requirements associated with these rights.

Take down policy

The Research Portal is Queen's institutional repository that provides access to Queen's research output. Every effort has been made to ensure that content in the Research Portal does not infringe any person's rights, or applicable UK laws. If you discover content in the Research Portal that you believe breaches copyright or violates any law, please contact openaccess@qub.ac.uk. 


\section{Length and thickness dependent optical response of liquid-exfoliated transition metal dichalcogenides}

Kevin Synnatschke, ${ }^{1}$ Patrick Cieslik, ${ }^{1}$ Andrew Harvey, ${ }^{2}$ Andres Castellanos-Gomez, ${ }^{3}$ Tian Tian, ${ }^{4}$ Chih-Jen Shih,${ }^{4}$ Alexey Chernikov, ${ }^{6}$ Elton J. G. Santos, ${ }^{5 *}$ Jonathan N. Coleman, ${ }^{2 *}$ Claudia Backes ${ }^{1 *}$

${ }^{1}$ Chair of Applied Physical Chemistry, Ruprecht-Karls University Heidelberg, Im Neuenheimer Feld 253, 69120 Heidelberg, Germany

${ }^{2}$ School of Physics and CRANN \& AMBER Research Centres, Trinity College Dublin, Dublin 2, Ireland

${ }^{3}$ Materials Science Factory. Instituto de Ciencia de Materiales de Madrid (ICMM-CSIC), Madrid, E-28049, Spain

${ }^{4}$ Institute for Chemical and Bioengineering, ETH Zürich, 8093 Zürich, Switzerland ${ }^{5}$ School of Mathematics and Physics, Queen's University Belfast, BT7 1NN, United Kingdom ${ }^{6}$ Department of Physics, University of Regensburg, Regensburg D-93040, Germany.

*E.Santos@qub.ac.uk

*colemaj@tcd.ie

*backes@uni-heidelberg.de

ABSTRACT: Due to their reduced dimensionality, 2D materials show intriguing optical properties and strong light matter interaction. In particular group VI transition metal dichalcogenides (TMDs) have been extensively studied and proof of principle optical applications demonstrated. Most studies to date focus on individual mono-or bilayered micromechanically-exfoliated samples which often display significant variations between flakes. In this work, we study size-dependent optical properties of four group VI TMD materials; $\mathrm{WS}_{2} ; \mathrm{MoS}_{2} ; \mathrm{WSe}_{2}$ and $\mathrm{MoSe}_{2}$, each consisting of ensembles of nanosheets suspended in the liquid environment. Samples were produced by liquid phase exfoliation and size-selected using cascade centrifugation with size and layer number distributions quantified by statistical atomic force microscopy. Differences in lateral size and layer number are 
reflected in systematic changes in the optical extinction and absorbance spectra, which we exploit to establish quantitative spectroscopic metrics to facilitate measurement of nanosheet dimensions for each of the four materials. The lowest energy resonance, referred to as Aexciton, is analyzed in more detail. In all cases, an exponential red-shift with increasing layer number is observed. Our experimental data, backed up with first principle calculations, reveals that the magnitude of the shift is dependent on the molecular mass of the central metal atom (W, Mo), while the rate at which the peak shifts from monolayer to bulk depends on the band gap of the semiconductor.

\section{Introduction}

Over the last decade, the study of 2-dimensional (2D) materials has become one of the most vibrant areas of nano-materials science. One of the most well-known families of 2D materials are the transition metal dichalcogenides (TMDs), which has over 40 members, including widely studied materials such as $\mathrm{MoS}_{2}$ and $\mathrm{WS}_{2}$. Many members of the TMD family are semiconducting ${ }^{1,2}$ and so are interesting for a number of application areas from optoelectronics to energy generation. ${ }^{3-11}$

Due to their reduced dimensionality, 2D materials in general, and TMDs in particular, exhibit remarkable physical properties; for instance, the group VI TMDs $\left(\mathrm{MoS}_{2}, \mathrm{WS}_{2}, \mathrm{MoSe}_{2}, \mathrm{WSe}_{2}\right)$ are direct bandgap semiconductors in the monolayer form, but display an indirect bandgap as few-layer and bulk materials. ${ }^{12}$ They show interband transitions in the visible and near-IR range with monolayers attractive for optoelectronic applications due to the possibility of efficient light emission. ${ }^{13,14}$ The optical properties of such 2D materials are dominated by tightly bound excitons with typical binding energies of several 100s of meV, considerably larger than their bulk counter parts. ${ }^{15}$ As a result, due to strong $2 \mathrm{D}$ confinement and reduced dielectric screening leading to small exciton radii, light-matter coupling is relatively strong compared to the majority of traditional inorganic semiconductors.

Although the electronic structure of TMDs has been studied quite heavily, a number of relatively basic properties are still not well-understood at a quantitative level. For example, the lowest energy optical transition in the absorbance spectrum, sometimes referred to as the optical gap, is excitonic in nature and represents the lowest excitation energy of the lowest energy exciton, the so-called A-exciton. Importantly, this resonance, $\mathrm{E}_{\mathrm{A}}$, is distinct from the free particle bandgap, $\mathrm{E}_{\mathrm{g}}$, which is considerably larger than the optical gap in 2D materials. 
The difference between these quantities is the exciton binding energy, $\mathrm{E}_{\mathrm{b}}: E_{g}=E_{A}+E_{b}$. Even today, the absolute values of $\mathrm{E}_{\mathrm{g}}$ and $\mathrm{E}_{\mathrm{b}}$ are not precisely known for all TMDs, especially for few-layered sheets, i.e. those thicknesses between monolayers and bulk. This is partially due to the presence of external contributions in the majority of studied samples stemming from environmental, doping, and strain inhomogeneities, and partially due to the lack of largenumber statistics.

Of the parameters introduced above, $\mathrm{E}_{\mathrm{A}}$ is easily observable in experiments, for example using linear absorbance or reflection spectroscopy as well as in photoluminescence (PL) and determines a number of key properties such as the onset of optical absorbance in the material. Interestingly, its evolution with changing layer thickness reflects several fundamental contributions. ${ }^{15}$ It is affected both by the hybridization of the electron states (i.e., essentially the conventional quantum confinement effect) and by the changes in the effective strength of the Coulomb interaction. The modification of the Coulomb interaction leads to the renormalization of both the freeparticle bandgap and exciton binding energy- effects which introduce shifts of similar magnitude, but opposite signs to $\mathrm{E}_{\mathrm{A}}$. The accurate determination of these quantities is thus very challenging, partially due to the inherent broadening of the relevant optical transition. However, interest in these parameters strongly motivates accurate thicknessdependent measurements of $\mathrm{E}_{\mathrm{A}}$, ideally involving large number statistics and performed across the family of semiconducting TMDs.

A number of papers have reported measurements of $\mathrm{E}_{\mathrm{A}}$ as a function of thickness for individual micromechanically-cleaved nanosheets via linear spectroscopy. ${ }^{12,}$ 16-20 However, such measurements may generally contain scatter and uncertainty due to the lack of ensemble averaging as well as factors such as strain and influence of the substrate in the individual flakes. As a result, it appears to be very useful to carefully investigate the thickness dependence of $E_{A}$ on large ensembles of nanosheets, ideally in a well-defined dielectric environment.

With this in mind, we suggest that nanosheets of known thickness distribution in the liquid phase may be an ideal model system fulfilling the above mentioned requirements. These can be prepared by liquid phase exfoliation (LPE) which is a widely-used technique for producing nanosheets from bulk layered crystals. LPE involves the production of $2 \mathrm{D}$ nanosheets by ultrasonication or shearing of layered materials in stabilizing liquids such as solvents or surfactant solutions. ${ }^{21-23}$ This method yields dispersions with nanosheet concentrations of $\sim \mathrm{gL}^{-}$ ${ }^{1}$ which are reasonably stable against aggregation. ${ }^{24-26}$ While this procedure initially gives 
nanosheets with broad size distributions, ${ }^{18,27}$ ensembles with large monolayer fractions can be also produced after size selection. ${ }^{17}, 18$ Due to its simplicity and scalability, ${ }^{22,}{ }^{28}$ LPE has been applied to a range of $2 \mathrm{D}$ materials including graphene, ${ }^{29-31} \mathrm{BN},{ }^{32,} 33 \mathrm{TMDs},{ }^{21}, 34-36 \mathrm{GaS},{ }^{37}$ InSe, ${ }^{38} \mathrm{SnS}^{39}$ or $\mathrm{GeTe}^{40}$ among others.

Recently, it has been demonstrated that high quality nanosheets dispersions can be produced by LPE which exhibit excitonic properties, such as narrow-linewidth photoluminescence, which are similar to that observed for micromechanically-cleaved TMDs. ${ }^{18}$ Such measurements have been enabled by improved size selection protocols, e.g. liquid cascade centrifugation (LCC), coupled with the ability to determine nanosheet size and thickness distributions accurately by statistical atomic force microscopy. ${ }^{18,27,41}$ The combination of these capabilities allows us to prepare sets of fractionated dispersions, where both nanosheet thickness and lateral size can be systematically varied. Such dispersions also contain very large numbers of nanosheets, such that spectroscopic measurements probe the ensemble, allowing reliable and reproducible measurements of average properties. In principle, such measurements can be used to study the fundamental physical properties of nanosheets in a high-throughput manner, for example by absorbance and PL spectroscopy. In addition, because every nanosheet is surrounded by the same solvent or surfactant coating, we would expect the dielectric environment experienced by the nanosheets to be quite uniform, ${ }^{42}$ rendering the influence of the environment essentially equal for all individual flakes in the solution.

Here, we fabricate dispersions of $\mathrm{MoS}_{2}, \mathrm{WS}_{2}, \mathrm{MoSe}_{2}$ and $\mathrm{WSe}_{2}$ nanosheets by LPE, before using LCC to generate factions with a range of well-defined and statistically quantified sizeand thickness-distributions, carefully confirmed by structural analysis techniques. For all four materials, we accurately measured the extinction, absorbance, scattering and photoluminescence spectra in dispersion for each size-selected fraction. We find systematic variations in the extinction and absorbance spectral shape with nanosheet lateral size, which we associate with edge effects. In addition, we find well-defined variations in the energy of the fundamental A-exciton transition, strongly correlated with changes to nanosheet thickness. In practical terms, these results provide reliable quantitative metrics to determine nanosheet lateral size and thickness from absorbance/extinction spectra. In addition, this data allows us to consistently study the scaling of electronic bandstructure and exciton energies with nanosheet thickness across a set of TMDs within the same experimental framework. 


\section{Results and Discussion}

Size selection and microscopic characterization of the TMD dispersions

In this work, we probe the lateral size- and thickness-dependence of the optical response of large populations of 2D nanosheets dispersed in liquids, focusing on the currently most prominent semiconducting TMD materials: $\mathrm{MoS}_{2}, \mathrm{WS}_{2}, \mathrm{MoSe}_{2}$ and $\mathrm{WSe}_{2}$. The advantage of this approach over an analysis of a few individual nanosheets (e.g. obtained by micromechanical cleavage) is that each optical measurement probes an ensemble, effectively averaging over very large numbers of nanosheets, typically $\sim 10^{10} \mathrm{~cm}^{-3}$. An initial challenge is that liquid-suspended nanosheets are inherently poly-disperse so that appropriate size selection and statistical size measurements are required. This will be described below with additional data shown in the SI (figures S1-10).

Polydisperse stock dispersions were produced by probe-sonicating commercially available TMD powders in an aqueous solution of the surfactant sodium cholate (SC, see methods and previous reports ${ }^{17,18}$ ). The as-obtained dispersions were then size-selected using liquid cascade centrifugation as described previously. ${ }^{18}$ In brief, a stock dispersion is first centrifuged at low centrifugal acceleration (expressed as relative centrifugal force in units of the earth's gravitational force, $g$, subsequently referred to as RCF or $g$-force,) to remove any pristine, nonexfoliated material in the sediment $(100 \mathrm{~g})$. The supernatant is then centrifuged at slightly higher RCF (400 g). A clear separation between sediment and supernatant is observed. The supernatant (containing smaller nanosheets) is decanted as completely as possible and the sediment (containing larger nanosheets) collected, re-dispersed in fresh aqueous SC, and retained for analysis. In turn, the supernatant is centrifuged at higher RCF (1,000 g). Again supernatant and sediment are separated, the sediment collected and the supernatant centrifuged at higher RCF. In this study, this process was continued until six fractions were collected in the sediments after each step. The final supernatant containing very small nanosheets $(<25 \mathrm{~nm})$ was discarded. Samples are denoted by indicating the upper and lower centrifugation boundary. For example, the sediment collected after centrifugation at $1,000 \mathrm{~g}$ of the supernatant obtained from the $400 \mathrm{~g}$ step is denoted as $0.4-1 \mathrm{k} g$.

The size-selected dispersions were diluted with water, drop-cast onto $\mathrm{SiO}_{2} / \mathrm{Si}$ wafers and subjected to statistical atomic force microscopy (AFM). Representative images of large (0.4$1 \mathrm{k} g$ ) and small $(10-22 \mathrm{k} g) \mathrm{MoSe}_{2}$ flake dispersions are shown in figure $1 \mathrm{~A}$ and $\mathrm{B}$, respectively. For each sample, 200-350 of such individually deposited nanosheets were analyzed and their 
length (L, longest dimension), width (W, perpendicular to L) and thickness recorded. To convert the measured apparent AFM thickness to layer number $(\mathrm{N})$, the AFM thickness is divided by $1.9 \mathrm{~nm}$ which is the apparent height of a surfactant-exfoliated TMD monolayer ${ }^{17}, 18$ as found by step height analysis. ${ }^{41}$ To correct the nanosheet length from AFM for cantilever broadening and pixilation, a subset of samples were subjected to transmission electron microscopy (TEM, Figure S7-S8) and mean nanosheet length of each dispersion from AFM and TEM compared. This resulted in a simple equation to correct the nanosheet length extracted from AFM (Figure S9).

After such corrections, histograms showing the distributions of nanosheet $\mathrm{L}$ and $\mathrm{N}$ are obtained, with examples shown for large and small MoSe2 nanosheets (Fig 1C, D). From such histograms, we can extract arithmetic means of layer number, $\langle\mathrm{N}\rangle$, and length, $\langle\mathrm{L}\rangle$, in each dispersion (all data see SI, Figures S1-6). However, since thinner nanosheets tend to be smaller in lateral size, as shown in previous reports, ${ }^{17,18}\langle\mathrm{~N}\rangle$ does not properly include the contribution of larger nanosheets. Thus, when a correlation to the spectroscopic data is targeted, it is more appropriate to calculate the mean layer number weighted by the volume fraction $\left(V_{f}\right)$ of each nanosheet. We term this weighted layer number, $\langle N\rangle_{V f}$ with the difference between this quantity and $\langle\mathrm{N}\rangle$ similar to the difference between weight-averaged and number-averaged molecular weight in polymer physics. The volume fraction weighted mean layer number can be found from the AFM data from nanosheet length (L), width (W) and thickness (i.e. layer number, N) using:

$\langle N\rangle_{V f}=\frac{\sum_{\text {all NS }} N^{2} L W}{\sum_{\text {all NS }} N L W}$

As shown in the SI (Figure S10), $\langle N\rangle_{V f}$ is closely correlated with $\langle\mathrm{N}\rangle$ for all four TMDs studied. This allows $\langle\mathrm{N}\rangle$ to be estimated from $\langle N\rangle_{V f}$ if required or vice versa.

To characterize the outcome of the size selection, we plot $\langle N\rangle_{V f}$ and $\langle\mathrm{L}\rangle$ as function of the midpoint of the centrifugal acceleration used in LCC in Fig 1 E, F for all size-selected dispersions. Interestingly, the data for all four martials fall roughly on a master curve for $\langle N\rangle_{V f}$ and $\langle\mathrm{L}\rangle$, despite the fact that these materials have significantly different densities. This suggests that the underlying separation mechanism in centrifugation is more complicated than 
simple mass fractionation, probably due to the high aspect ratio of the nanosheets. Both $\langle N\rangle_{V f}$ and $\langle\mathrm{L}\rangle$ fall as power-laws with the central $g$-force: $\langle N\rangle_{V f} \propto\left(g-\right.$ force $^{-0.38}$ and $\langle L\rangle \propto(g-\text { force })^{-0.4}$.

In addition to mean nanosheet layer number and length, AFM can be used to determine the volume fraction of monolayers $\left(V_{f, M L}\right)$ in a dispersion. Note that, since the monolayer population is low in samples extracted at low centrifugal acceleration, a significant number of AFM counts $(>300)$ is required. In figure $1 \mathrm{G}$, we plot the monolayer volume fraction as function of the midpoint of the centrifugal acceleration. Again, we find the data from all four TMDs to collapse on the same curve supporting the robustness of the size selection and the accuracy of the AFM statistical analysis. We find the monolayer volume fraction to increase with central acceleration before eventually leveling off (empirical function: $V_{f, M L}=\left[1.2+70(g-\text { force })^{-1.3}\right]^{-1}$. Clearly, we would expect $V_{f, M L}$ to scale with $\langle N\rangle_{V f}$. In figure $1 \mathrm{H}$, we plot the monolayer volume fraction as function of $\langle N\rangle_{V f}$ finding a well-defined empirical correlation, consistent with:

$V_{f, M L}=\left[0.9+0.1\langle N\rangle_{V f}^{3.4}\right]^{-1}$

Eq 2

Thus, equation 2 allows the monolayer volume fraction to be estimated from $\langle N\rangle_{V f}$ (or arithmetic mean $\langle\mathrm{N}\rangle$ ) for any of the four TMDs under study. This is extremely useful given that the latter parameter can be extracted more easily from AFM statistics and, as we shall see below, can be determined from spectroscopic data.

\section{Size dependent optical extinction spectra}

The simplest optical response for which we might expect size-dependent effects is extinction spectroscopy. Indeed, size-dependent changes to extinction coefficient spectra have been observed for a number of 2D materials. ${ }^{17,18,24,37,43-46}$ Here, extinction spectra were measured for all size-selected dispersions of each of the four TMDs. These spectra were converted to extinction coefficient, $\varepsilon$, spectra using the nanosheet concentration, $C$, as determined by drying and weighing (the extinction coefficient is defined via the transmittance, $T$ via $T=10^{-\varepsilon C l}$, where $l$ is the cuvette thickness). For example, the extinction coefficient spectra for the sizeselected $\mathrm{MoSe}_{2}$ dispersions are shown in figure 2A (see SI figures S11-17 for all spectra). The 
spectra show the signatures of excitonic transitions associated with TMDs. ${ }^{2,47}$ In particular, the fundamental A-excitonic resonance (e.g. at $\sim 790 \mathrm{~nm}$ for $\mathrm{MoSe}_{2}$ ), is associated with transitions at the K-point in the Brillouin zone. Importantly, this energy is generally lower than the corresponding free-particle bandgap transition by an amount equal to the exciton binding energy as described in the introduction. In line with previous observations, for each TMD, the position of this peak varies over the fractions (figure $2 \mathrm{~A}$ inset), as will be discussed in detail below. In addition, it is clear that the extinction coefficient spectra systematically vary with nanosheet size.

When measuring size-dependent optical responses, it is important to realize that for nanoparticle suspensions, extinction contains contributions from light scattering as well as absorbance. ${ }^{17,18,48,49}$ Scattering is particularly significant for large nanosheets in the highwavelength regime where absorbance is negligible. ${ }^{17}$ This is clear for the fraction containing the largest nanosheets in figure $2 \mathrm{~A}$, where scattering contributes strongly to the optical response at energies below the A-exciton resonance. We illustrate this further in figure $2 \mathrm{~B}$, where we plot the extinction coefficient at the A-exciton as function of nanosheet lateral size for the four TMDs. The extinction coefficient remains largely invariant for $\langle\mathrm{L}\rangle$ below $\sim 150$ $\mathrm{nm}$ before it increases steeply due to scattering. However, it has been shown that the scattering spectra of liquid-exfoliated TMDs roughly follow the absorbance spectra in shape in the resonant regime. ${ }^{17}$ This allows us to, in principle, extract any size-dependent information encoded in the absorbance from the extinction spectra, albeit with some restrictions in terms of peak position as discussed below.

\section{Dependence of spectral profile on nanosheet length}

From the data in figure $2 \mathrm{~A}$, it is clear that extinction coefficients at low wavelengths (high energy) decrease with increasing nanosheet size. This behavior was previously attributed to differences in electronic structure between nanosheet edges and center regions, resulting in distinct absorbance coefficients at nanosheet edge versus basal plane. ${ }^{17}$ Coupled with lengthdependent scattering effects, ${ }^{50}$ such effects can lead to strongly length-dependent extinction coefficients as shown in figure $2 \mathrm{C}$, where the extinction coefficient at $250 \mathrm{~nm}$ is plotted as function of $\langle\mathrm{L}\rangle$ for the four TMDs. In all cases, the extinction coefficient at $250 \mathrm{~nm}$ falls with increasing nanosheet size. This size dependence of the extinction coefficient is of great practical use because it allows mean nanosheet length to be extracted from extinction spectra as previously shown for $\mathrm{MoS}_{2}$ and $\mathrm{WS}_{2} .{ }^{17}, 18 \mathrm{We}$ demonstrate this in figure 2D by plotting the 
ratio of extinction measured at two wavelengths ( $\lambda_{1}$ and $\lambda_{2}$, see legend) versus the mean nanosheet length measured by AFM. Applying a simple theoretical model ${ }^{17}$ to describe edge effects, we expect extinction intensity ratios to scale with $\langle\mathrm{L}>$ according to equation 3 :

$$
R=\frac{\operatorname{Ext}\left(\lambda_{1}\right)}{\operatorname{Ext}\left(\lambda_{2}\right)}=\frac{A_{1}\langle L\rangle+B_{1}}{A_{2}\langle L\rangle+B_{2}}
$$

where the parameters $\mathrm{A}$ and $\mathrm{B}$ are obtained from fitting and are given in table $1\left(\mathrm{~B}_{2}\right.$ was fixed at $1 \mathrm{~nm}$ in all cases to reduce the number of fit parameters). This equation quantitatively links the nanosheet lateral size to the spectra profile and allows mean nanosheet lengths to be estimated from extinction spectra for any of these TMD materials. Note that using peak intensity ratios has the advantage that spectra can be measured in arbitrary units on the y-axis), i.e. without knowledge of nanosheet concentration.

\section{Dependence of the exciton transition energies on the nanosheet thickness}

As described in the introduction, we expect both the free-particle bandgap and the exciton binding energy to scale with the nanosheet thickness leading to a dependence of the A-exciton energy on $\langle\mathrm{N}\rangle$. In the following, we discuss these thickness effects and show that they provide access to fundamental processes associated with the intrinsic changes of the interlayer electronic hybridization and dielectric screening effects with the number of layers. The inset in figure $2 \mathrm{~A}$ shows that the positions of excitonic transitions shift across the size-selected TMD dispersions (example of $\mathrm{MoSe}_{2}$ shown here, all TMDs see SI figures S12-17). Such shifts were previously observed for $\mathrm{LPE} \mathrm{WS}_{2}$ and $\mathrm{MoS}_{2}$ and ascribed to variations in nanosheet thickness via confinement effects, although the exact origin was not described in detail. ${ }^{17,}{ }^{18}$ In the following, we discuss such nanosheet thickness effects on the exciton energy for the four TMDs under study: $\mathrm{WS}_{2}, \mathrm{MoS}_{2}, \mathrm{WSe}_{2}$ and $\mathrm{MoSe}_{2}$.

First, we need to consider the contribution of light scattering to the extinction spectra which cannot be neglected in dispersions containing larger/thick nanosheets. Transmission, and so extinction measurements, include contributions from both absorbance and scattering of light such that the extinction coefficient is the sum of absorbance and scattering coefficients. ${ }^{17}$ In the nonresonant regime, i.e., for photon energies below the onset of optical absorbance, the contribution from scattering is dominant and follows a powerlaw in wavelength as described in detail elsewhere. ${ }^{50}$ Such powerlaw behavior is observed in the extinction spectra of all TMD fractions isolated at low centrifugal acceleration (see SI, Figures S11-S17) at wavelengths above that of the A-exciton. In the resonant regime, the scattering is red-shifted to the 
absorbance and thus can have an impact on the apparent positions of excitonic transitions in extinction spectra. ${ }^{17}$

To isolate the effect of scattering, optical spectra were acquired in the center of an integrating sphere, where scattered light is collected and therefore absorbance (Abs) measured. ${ }^{17}$ To illustrate that scattering contributes to the extinction spectra in samples containing larger/thicker nanosheets, the extinction/absorbance/scattering coefficient spectra of the largest fraction of $\mathrm{MoSe}_{2}(0.1-0.4 \mathrm{k} g)$ are shown in figure $3 \mathrm{~A}$. In this particular sample, the scattering coefficient is larger than the absorbance coefficient.

Although excitonic peaks measured on ensembles (e.g., the A-exciton peak in figure 2A) appear as single resonances, they can contain information about the nanosheet thickness distribution within the ensemble and represent a weighted average of all nanosheets in a given dispersion. ${ }^{18}$ As a result, such peaks are best analyzed via their second derivative, as double differentiation results in a significant narrowing $(\sim \times 1 / 3)$ of any component peaks. This can allow for the separation of individual contributions in case of sufficiently large spectral shifts, especially for samples with high mono- to few-layer fraction. ${ }^{18}$ In addition, the background from scattering or partial overlap with the B-exciton - is essentially eliminated, allowing for a more precise determination of the shifts in the peak positions even without more comprehensive data fitting that can be error-prone due to the uncertainty in number of components. The second derivative in the A-exciton region in figure $3 \mathrm{~B}$ further shows the effect of scattering on the A-exciton peak position: In the absorbance spectrum, the A-exciton is observed at $1.542 \mathrm{eV}$, while it is red-shifted to $1.502 \mathrm{eV}$ in the scattering spectrum, with the value for extinction being in between. While, on a practical note, such shifts are small, they will have an impact when it comes to understanding the nature of the excitonic shifts. Therefore, we only discuss absorbance spectra in the following, with data from extinction (which is practically more useful in terms of metrics for layer number) shown in the SI.

In figure $3 \mathrm{C}$ the second derivative of the absorbance in the A-exciton region of the four TMDs under study is displayed for fractions containing thick $(0.4-1 \mathrm{k} g)$ and thin $(10-22 \mathrm{k} g)$ nanosheets. In each case, the A-exciton is significantly red-shifted in the dispersions containing thick nanosheets (thin lines) compared to the monolayer-rich counterparts (thick lines). Only in the case of $\mathrm{WS}_{2}$ do we observe a well-resolved fine-structure in the spectrum associated with the dispersion of thin nanosheets. Such fine structure is attributed to contributions from monolayers, as well as few-layers to the spectrum. ${ }^{18}$ That such splitting is clearly observed for 
$\mathrm{WS}_{2}$ but not the other TMDs can be attributed to the inherently narrower linewidths in $\mathrm{WS}_{2}$ as described below. The position of the peaks in the $\mathrm{d}^{2} \mathrm{Abs} / \mathrm{dE}^{2}$ curves of the monolayer-rich samples are given in table 2.

In addition, photoluminescence measurements (excitation $430 \mathrm{~nm}$ ) were performed on the most monolayer-rich dispersions (see table 2 for mean nanosheet thicknesses and monolayer contents). For additional PL data see SI, figure S18. For all four TMDs, the PL can be fit well to a single Lorentzian suggesting that predominantly excitonic emission is observed with negligible contributions from trions, consistent with reasonably small doping in the studied systems. ${ }^{51}$ The PL peak positions and full width at half maximum (FWHM) values are summarized in table 2 and compared with the absorbance positions extracted from the second derivative spectra in figure 3C. As is also observed for micromechanically-cleaved TMDs the room-temperature line-width is narrowest for $\mathrm{WS}_{2}$ and is somewhat larger for $\mathrm{MoSe}_{2}, \mathrm{MoS}_{2}$, and $\mathrm{WSe}_{2}{ }^{52}$ This observation is consistent with previous experimental and theoretical findings ${ }^{53}$ demonstrating that, at elevated temperatures such as room temperature, the exciton linewidth is dominated by exciton-phonon scattering in samples with sufficiently small inhomogeneous broadening. While a number of potential pathways contribute to excitonphonon interaction, the coupling of A-excitons to low-energy acoustic phonons in particular is found to be much weaker in $\mathrm{WS}_{2}$. Among the reasons behind this are lower effective masses of the electrons and holes ${ }^{54}$ combined with smaller deformation potential matrix elements. ${ }^{55}$ This results in significantly reduced linewidths, both in PL and absorbance, for $\mathrm{WS}_{2}$ compared to the other TMDs.

For $\mathrm{WS}_{2}\left(\langle N\rangle_{V f}=1.6\right)$, the PL position $(2.032 \mathrm{eV})$ coincides closely with the higher energy monolayer peak of the corresponding $\mathrm{d}^{2} \mathrm{Abs} / \mathrm{dE}^{2}$ curve $(2.034 \mathrm{eV})$. This is implies a minor Stokes-shift ( $\sim 2 \mathrm{meV})$. However, for the other three TMDs, especially for the selenides, the Stokes shifts are not negligible. For example, the $10-22 \mathrm{k} g \mathrm{WSe}_{2}$ dispersion with a monolayer volume fraction of $\sim 30 \%$ and $\langle N\rangle_{V f}=2.3$ has an A-exciton absorbance at $1.664 \mathrm{eV}$ with PL centered at $1.657 \mathrm{eV}$. This implies a Stokes shift of $7 \mathrm{meV}$ which is still a lower estimate given the absorbance contains a $\sim 70 \%$ contribution from few-layers which redshifts the observed absorbance peak relative to the true monolayer-only peak, whereas only the monolayer is detected in PL. Similarly, in spite of only $<30 \%$ of the nanosheets being monolayer in the monolayer-richest $\mathrm{MoSe}_{2}$ dispersion, the PL (only monolayers) is red-shifted to the absorbance by $4 \mathrm{meV}$. Since the absorbance peaks are averaged over all nanosheet thicknesses in the 
particular samples, this implies a much larger Stokes shift for the selenides compared to $\mathrm{WS}_{2}$. In the $\mathrm{MoS}_{2}$ sample, the photoluminescence is blue-shifted compared to the absorbance resonance. This can only be understood if the actual Stokes shift of monolayer PL to monolayer absorbance- a value that is not directly accessible- is small. Taken together, no quantitative information on the monolayer absorbance is directly accessible in any case except $\mathrm{WS}_{2}$, where the monolayer and few-layer transitions resulting in splitting of the A-exciton peak are separately observed due to smaller linewidths of the exciton resonance.

In addition to producing monolayer-enriched samples, LPE in combination with size selection, allows us to obtain fractions with varying thickness distributions. This enables a consistent and statistically meaningful analysis of the A-exciton transition energy (from absorbance spectra) with mean layer number for nanosheet ensembles (from AFM). This data is plotted in figure 3E-H for the four TMDs under study. In all cases, we see a fall-off in exciton position towards lower energies with increasing nanosheet thickness with reductions of 32-65 meV observed, consistent with previous data. ${ }^{16-18}$ We have also included a comparison with literature data (reflectance spectroscopy) for individual micromechanically-cleaved nanosheets (although the precise origin of the thickness dependent shifts is rarely discussed in detail). ${ }^{12,} 19,20$ While the literature data is more scattered than the LPE data, probably due to sheet-to-sheet variations, it agrees reasonably well with our observations in large ensembles. Here, we further emphasize that our approach of measuring ensembles in liquids suppresses individual sampling error due to its inherent averaging. However, we note that some literature data obtained from microreflectance spectra on micromechanically exfoliated nanosheets ${ }^{16}$ do displays deviations from our data (analyzed and discussed in detail in the SI, Figure S19-22). The main sources of discrepancy are related to differences in sample type (potentially strain, intercalation), as well as type of measurement (see SI section 2.3).

In general, we find the decrease in A-exciton position with increasing layer number (figure 3E$\mathrm{H})$, to be consistent with an exponential decay of the form:

$$
E_{A}=E_{A, B u l k}+\left(E_{A, M L}-E_{A, B u l k}\right) e^{-\left(\langle N\rangle_{V f}-1\right) / N_{0}}
$$

where $E_{A}$ is the measured exciton energy, $E_{A, b u l k}$ is the exciton energy of the bulk layered crystal, $\mathrm{E}_{\mathrm{AML}}$ is the exciton energy of a monolayer and $\mathrm{N}_{0}$ is a characteristic, phenomenological decay constant describing the rate at which the monolayer A-exciton energy approaches the values for the bulk with increasing layer number. Fit parameters obtained by fitting equation 4 to the data in figure $3 \mathrm{E}-\mathrm{H}$ are given in table 3 . We note that this empirical fitting procedure is 
not restricted to TMDs, but can be potentially applied to any exfoliated 2D materials where excitonic shifts with layer number are observed.

Firstly, as illustrated in figure $4 \mathrm{~A}$, we find that the values of $\mathrm{E}_{\mathrm{A}, \mathrm{Bulk}}$ extracted by fitting the curves in figure $3 \mathrm{E}-\mathrm{H}$ match well to the equivalent data extracted from literature (see Ref. ${ }^{52}$, 56,57 ). It is challenging to compare the values of $\mathrm{E}_{\mathrm{A}, \mathrm{ML}}$ extracted from the fits to literature values, due to the significant differences in dielectric environment between individual monolayers on a substrate (as generally measured in the literature) and our surfactant-coated monolayers. In addition, other than for $\mathrm{WS}_{2}$, we cannot use our absorbance data to infer a ML energy due to the lack of observable mono/few-layer splitting. As a result, we must compare the fit data to the PL line positions reported above. As shown in figure $4 \mathrm{~B}$, the values of $\mathrm{E}_{\mathrm{A}, \mathrm{ML}}$ extracted by fitting the curves in figure 3E-H match well to the positions of the PL peaks shown in figure 3D except for larger Stokes shifts of the selenides compared to the sulfides as described above. Overall, this supports the validity of the data fitting and suggests that limited data sets might best fit by fixing $\mathrm{E}_{\mathrm{A}, \text { bulk }}$ and/or $\mathrm{E}_{\mathrm{A}, \mathrm{ML}}$ using literature values.

The difference between $E_{\mathrm{A}, \mathrm{ML}}$ and $\mathrm{E}_{\mathrm{A}, \mathrm{Bulk}}$ is plotted for each material in figure 4C. This parameter represents the entire range over which the exciton position can vary for different nanosheet thicknesses. Interestingly the values of this parameter are very similar for the Wbased compounds at 67 and $65 \mathrm{meV}$ (WS 2 and $\mathrm{WSe}_{2}$ respectively) while the Mo-compounds are also similar at 49 and $56 \mathrm{meV}$ (for $\mathrm{MoS}_{2}$ and $\mathrm{MoSe}_{2}$ respectively). Furthermore, as plotted in figure $4 \mathrm{D}$, we find the decay constant, $\mathrm{N}_{0}$, to scale roughly linearly with $\mathrm{E}_{\mathrm{A}, \mathrm{Bulk}}$ as obtained from the LPE fits.

To understand these observations in more detail, it is worth considering the factors which control the energetic position of the A-exciton. In general, the energy of an excitonic resonance is determined by the energy of the free-particle bandgap, $\mathrm{E}_{\mathrm{g}}$, corresponding to the onset of unbound electrons and holes, minus the excitonic binding energy, Eb. ${ }^{15}$ Due to sizable dielectric screening effects in bulk, the exciton binding energy tends to be relatively small in 3D materials, with bulk $\mathrm{MoS}_{2}$, for example, showing $\mathrm{E}_{\mathrm{b}} \sim 50 \mathrm{meV}$ (figure $4 \mathrm{E}$ ). ${ }^{58}$ However, in confined systems such as 2D nanosheets or 1D nanotubes, exciton binding energies can be considerably larger than in 3D systems. ${ }^{15}$ For $2 \mathrm{D}$ systems, quantum confinement of the excitons in one layer would contribute a factor of 4 to the binding energy in comparison to a full 3D hydrogenic model, even though in bulk, the excitons may be largely localized within a single layer already (as discussed in the early literature).${ }^{59}$ An additional, and arguably the strongest, contribution arises due to reduced dielectric screening of the environment as the layer number 
decreases, strongly enhancing the effective strength of the Coulomb interaction between charge carriers. Stronger Coulomb interaction leads in turn both to an increase of the exciton binding energy and to a shift of the free-particle bandgap transition on the similar order of magnitude ${ }^{15}$ as the thickness changes from bulk to monolayer. While the absolute peak position of the exciton resonance $\mathrm{E}_{\mathrm{A}}$ is relatively simple to measure in experiment using a range of techniques, both $E_{g}$ and $E_{b}$ are significantly harder to obtain, typically requiring either independent knowledge of the free-particle bandgap, e.g., through tunneling spectroscopy ${ }^{60}$ or the observation of higher excited states. ${ }^{61}$ The reported literature data for transition metal dichalcogenides is somewhat scattered due to both challenges associated with the interpretation of the experiments, but also due to inter-sample variations, in particular with respect to differences in static dielectric screening and potential contributions from residual doping. ${ }^{15} \mathrm{It}$ appears reasonable, however, that the exciton binding energies should be on the order of 0.5 $0.6 \mathrm{eV}$ in freestanding samples, ${ }^{62}$ yet can be significantly smaller due to additional environmental screening when placed on substrates or, by analogy, in the presence of surfactants. ${ }^{63,64}$

For quasi-2D materials such as few-layer nanosheets, both $E_{g}$ and $E_{b}$ are known to scale with nanosheet thickness, N. However, the resulting absolute change of the fundamental optical transition is a non-trivial combination of several contributions. First, the free-particle bandgap is determined by the inter-layer hybridization of the respective electronic states, essentially mirroring the quantum confinement effect observed in III-V and II-VI quantum well systems. ${ }^{65}$ In semiconducting TMDs these effects can be indeed relatively strong, e.g., for $\Gamma$ and $\Lambda / Q / \Sigma$ valleys due to the significant contributions of the chalcogen orbitals at the layer surface to the respective electronic wavefunctions. This leads to sizable overlap integrals from the respective states in neighboring layers. The electron wavefunctions at the K-points of the Brillouin zone, that determine the onset of optical absorbance, however, are largely localized at the metal atoms in the middle of the individual layers. Thus, they couple only weakly to the proximate layers, as discussed extensively in the literature. ${ }^{12,66}$ Nevertheless, even a small finite coupling would contribute to the shift of the bandgap to lower energies with increasing layer thickness. Secondly, the effective strength of the Coulomb interaction also changes with the layer number, largely due to the changes in the environmental screening of the individual layers. The value of the screening itself depends both on the dielectric permittivity of the nanosheet and its surroundings, and is therefore also distance dependent. Importantly, the overall efficiency of the dielectric screening increases with the number of layers and leads both to shift of the free- 
particle bandgap $\mathrm{E}_{\mathrm{g}}$ towards lower energies and to a decrease of the exciton binding energy $\mathrm{E}_{\mathrm{g}}$. While the two effects partially cancel each other out, a remaining shift of the resulting absolute transition energy can remain. Thus, the thickness dependence of both $\mathrm{E}_{\mathrm{g}}$ and $\mathrm{E}_{\mathrm{b}}$ will generally lead to a thickness dependence of the exciton energy, $\mathrm{E}_{\mathrm{A}}$, observed figure $3 \mathrm{E}-\mathrm{H}$ :

$$
E_{A}(N)=E_{g}(N)-E_{b}(N)
$$

However, what is particularly interesting is the scale of the thickness variation of each quantity. As shown schematically in figure $4 \mathrm{~F}$, for a material like $\mathrm{MoS}_{2}$, both $\mathrm{E}_{\mathrm{g}}$ and $\mathrm{E}_{\mathrm{b}}$ vary by $\sim 300$ $400 \mathrm{meV}$ going from monolayer to bulk. However, for the same material $\mathrm{E}_{\mathrm{A}}$ varies by only 30$40 \mathrm{meV}$ over the same thickness range. Because of this small variation, the $\mathrm{E}_{\mathrm{A}}$ versus $\langle\mathrm{N}\rangle$ curves such as those in figure 3E-H are almost flat compared to the much larger expected variations of $E_{g}$ and $E_{b}$.

Our detailed observations and the resulting trends should be thus considered within this general framework of changes in the electron hybridization and Coulomb effects. Considering the latter, we can understand an important part of the contributions to the dependence of $\mathrm{N}_{0}$ on $E_{A, b u l k}$ presented in figure $4 \mathrm{D}$, from the Moss relation - a higher bandgap results in lower intrinsic materials permittivity. For higher bandgaps, this would directly result in a smaller contribution of the nanosheet permittivity to the effective dielectric screening and thus in a smaller contribution of the Coulomb effects to the thickness dependence. That would in turn lead to a larger value of the phenomenological decay constant, $\mathrm{N}_{0}$, consistent with figure 4D. The microscopic origin of this effect is discussed in more detail in the following section.

\section{Theoretical description of the exciton shifts}

To verify our proposed mechanism of the thickness-dependent exciton peak energy, we quantitatively investigate the evolution of both $\mathrm{E}_{\mathrm{g}}(\mathrm{N})$ and $\mathrm{E}_{\mathrm{b}}(\mathrm{N})$ using first principles methods (See Methods for details). Due to the large computational effort required for the simulations of the many-body excitonic properties for several layers $(\mathrm{N}>10)$ into the systems, full-scale $\mathrm{G}_{0} \mathrm{~W}_{0}{ }^{67}$ and $\mathrm{G}_{0} \mathrm{~W}_{0}-\mathrm{BSE}^{68}$ methods become impractical. A few approximation methods are developed to explain the evolution of quasi-particle bandgap ${ }^{69}$ and exciton binding energy ${ }^{63}$ of 2D materials due to influence of dielectric environment, which indicates the evolution of $E_{\mathrm{g}}(N)$ and $E_{\mathrm{b}}(N)$ might be unified by the same theoretical framework taking dielectric screening into account. Inspired by this, we use the recently developed quantum electrostatic heterostructure (QEH) approach. ${ }^{70,71}$ The QEH model assumes the dielectric screening of multilayer 2D material stack, which is characterized by the generally spatially (or momentum) dependent 
dielectric function $\varepsilon^{-1}$, can be represented by the density response function $\chi_{i}$ of the individual 2D layers. Due to the fact that interlayer excitons usually have smaller binding energy compared with the intralayer exciton, ${ }^{72}$ we only calculate the latter exciton binding energy, which is obtained by solving the $2 \mathrm{D}$ Schrödinger equation ${ }^{3}$ :

$$
\left[-\frac{\nabla^{2}}{2 m_{e x}^{*}}+W_{N}(r)\right] F(r)=E F(r) \quad \text { Eq } 6
$$

where $m_{e x}^{*}$ is the in-plane effective mass of the exciton, $E$ is the eigenenergy, $F(r)$ is the wave function of the exciton, and $W_{N}(r)=-\varepsilon^{-1} e^{2} / r$ is the screened Coulomb interaction energy inside a 2D stack of $N$ layers, which is further computed by the QEH model. Conversely, dielectric screening is also found to influence the bandgap of $2 \mathrm{D}$ materials, both in experiments ${ }^{73}$ and theory. ${ }^{74}$ To simulate the evolution of the quasiparticle eigenenergy caused by the dielectric screening of multilayers, we adapted the $\mathrm{G} \Delta \mathrm{W}-\mathrm{QEH}$ model which is shown to correctly capture the change of band structure of 2D stacks and heterostructures, ${ }^{70,75}$ which corrects the eigenenergies from $\mathrm{G}_{0} \mathrm{~W}_{0}$ calculation by the degree of screening:

$$
E_{n, k}^{i, N}=E_{n, k}^{i}\left(G_{0} W_{0}\right)+\Delta E_{n k}^{i, s c r}
$$

where $E_{n, k}^{i, H S}$ is the eigenenergy of layer $i$ in the N-layer stack, $E_{n, k}^{i}\left(G_{0} W_{0}\right)$ is the eigenenergy for layer $i$ from $\mathrm{G}_{0} \mathrm{~W}_{0}$ calculations, and $\Delta E_{n k}^{i, s c r}$ is the correction due to dielectric screening. The G $\Delta \mathrm{W}-\mathrm{QEH}$ model is shown to effectively describe the dielectrically-renormalized quasiparticle bandgap of $2 \mathrm{D}$ materials at the $\mathrm{K}$ point, where the effect of hybridization on the bandgap is minimal ${ }^{75}$. The $\mathrm{E}_{\mathrm{g}}(\mathrm{N})$ and $\mathrm{E}_{\mathrm{b}}(\mathrm{N})$ values are therefore obtained from $\mathrm{G} \Delta \mathrm{W}-\mathrm{QEH}$ and QEH models, respectively, using the ab initio package GPAW. ${ }^{76}$

To test the models, we first calculate the band structures of the $2 \mathrm{D}$ materials studied in the experiments. As shown in Figure 5A, G $\Delta \mathrm{W}-\mathrm{QEH}$ model predicts that when increasing the layer number $N$ (color from violet to red), the eigenenergies of the CBs are shifted towards lower energy near the K-point, thus reducing the bandgap $E_{g}(N)$. Furthermore, we superimpose the position of the A exciton energy $E_{A}(N)$ schematically as horizontal lines below the $C B$ band for illustration purposes, as calculated using Eq. (5). For better clarity, only the lowest/highest bands in the $\mathrm{CB} / \mathrm{VB}$ are included for every multilayer system, and the energy maxima of VB are aligned. As can be seen, the changes of the absolute energy of the exciton ground state transitions $\mathrm{E}_{\mathrm{A}}(\mathrm{N})$ is significantly smaller than that of the shifts in the quasiparticle band structure and, in particular, the bandgap $\mathrm{E}_{\mathrm{g}}(\mathrm{N})$, which is in agreement with the experimental 
results. We then take a closer look at the magnitudes of $\mathrm{Eg}_{\mathrm{g}}(\mathrm{N})$ and $\mathrm{Eb}(\mathrm{N})$ as calculated by the QEH models. As shown in Figure $5 \mathrm{~B}$, both $\mathrm{Eg}_{\mathrm{g}}(\mathrm{N})$ (circles) and $\mathrm{Eb}_{\mathrm{b}}(\mathrm{N})$ (triangles) show similar trends of decrease with increasing layer number $\mathrm{N}$ : the differences between $\mathrm{E}_{\mathrm{g}}(\mathrm{N}=1)$ and $\mathrm{E}_{\mathrm{g}}(\mathrm{N}=\infty)$ are about $0.4 \sim 0.5 \mathrm{eV}$, and the differences between $\mathrm{E}_{\mathrm{b}}(\mathrm{N}=1)$ and $\mathrm{E}_{\mathrm{b}}(\mathrm{N}=\infty)$ are about $\sim 0.35 \mathrm{eV}$. Notably, the results of $\mathrm{MoS}_{2}$ agree with those reported by Cho et al. ${ }^{69}$ using electrostatically-renormalized electronic and optical gaps. The trends of both $E_{g}(N)$ and $E_{b}(N)$ can be well-fitted using the exponential model as in Eq. (4), giving the characteristic decay length $\mathrm{N}_{0}$, as defined in Eq. (4), to be 3.5 for $\mathrm{E}_{\mathrm{g}}$ and 2.2 for $\mathrm{E}_{\mathrm{b}}$, indicating the influence of layerdependent dielectric screening is quite similar for both electronic and optical gaps. The Aexciton transition energy calculated by the QEH models is shown in Figure 5C (squares) as compared with that obtained by experiments (stars). From the QEH models, the layerdependent variation of $\mathrm{E}_{\mathrm{A}}$ is reduced to $120 \sim 150 \mathrm{meV}$ (Figure 5D) as compared with that of $\mathrm{E}_{\mathrm{g}}$ and $\mathrm{Eb}$, confirming the mechanism illustrated in Figure 4. We note that the degree of variation predicted by the model is still larger than that experimentally observed, which may be explained by additional environmental dielectric screening ${ }^{77}$ via the solvents, that gives a close agreement with the measurements (inset in Fig. 5C). Finally, we compare the value of $\mathrm{N}_{0}$ compared with that obtained from fitting of the experimental data, as shown in Figure 5E. Exponential fitting of the simulated $\mathrm{E}_{\mathrm{A}}(\mathrm{N})$ magnitudes gives $\mathrm{N}_{0} \sim 6$ (stars), which is larger than that using the experimental data, indicating the experimental observed exciton decay is faster than from the model, which may again be caused by the environmental screening of the solvent. However, as $\mathrm{Eb}(\mathrm{N})$ is considered instead both theory and experiments agreed closely. In summary, although minor numerical discrepancy exists, our model is able to capture the experimentally observed layer-dependent evolution of exciton peak, and confirms the proposed mechanism of the combined effect of layer-dependent scaling of both bandgap and binding of 2D material stacks. Furthermore, the evolution of both $E_{g}(N)$ and $E_{b}(N)$ can be unified by the layer-dependent dielectric screening, which provides a versatile approach to study Coulomb interaction effects in other low-dimensional materials.

\section{Conclusion}

In summary, we have measured optical extinction/absorbance/scattering and photoluminescence spectra of four group VI TMDs ( $\left.\mathrm{WS}_{2}, \mathrm{MoS}_{2}, \mathrm{WSe}_{2}, \mathrm{MoSe}_{2}\right)$. In contrast to most reports in literature to date which focus on micromechanically-exfoliated samples, nanosheets in the liquid phase were analyzed. This bears the advantage that an ensemble of 
sheet in the order of $10^{10}$ nanosheets are investigated in each measurement. In addition, the dielectric environment, i.e the surrounding liquid is quite homogeneous. As such, sample inhomogeneities from varying defect contents, inhomogeneous dielectric environment, strain, doping etc. that arise in flake by flake measurements of micromechanically cleaved samples are inherently averaged out. As such, even subtle differences in the layer number dependent optical response for few-layered nanosheets are accessible.

To correlate the size-dependent optical response to the nanosheet dimensions, size distributions for each TMD were narrowed by size selection using liquid cascade centrifugation. This yielded $\sim 6$ size-selected fractions for each TMD. Using recent advances in size quantification by statistical atomic force microscopy, ${ }^{27}$ lateral size, $\mathrm{L}$ and layer number, $\mathrm{N}$ distributions were obtained. This size information was correlated to the optical spectra. Both lateral size and nanosheet layer number are reflected in well-defined changes in optical absorbance/extinction/scattering spectra. Practically speaking, this enables us to establish quantitative spectroscopic metrics for concentration, $\langle\mathrm{L}\rangle$ and $\langle\mathrm{N}\rangle$ for the four TMDs under study that can be used to determine these quantities accurately from optical spectra of unknown samples without microscopy statistics.

Probably the most interesting change in optical spectra is a small, but systematic blue-shift of the A-exciton with decreasing layer number across the four TMDs. This shift arises from two effects of similar magnitude, but opposite sign: changes in the electronic bandgap, as well as exciton binding energy. Our experimental data, backed up by first principle calculations, shows that the magnitude of the shift of the A-exciton energy is larger for $\mathrm{WS}_{2}$ and $\mathrm{WSe}_{2}$ compared to $\mathrm{MoS}_{2}$ and $\mathrm{MoSe}_{2}$, i.e. depends on the molecular mass of the metal atoms. In contrast, the rate at which the A-exciton energy shifts from the monolayer to bulk value is widely dependent on the materials' bandgap at the associated point in the Brillouin zone.

To conclude, this work provides useful quantitative metrics for nanosheet concentration and dimensions from readily accessible optical extinction/ absorbance spectra. In addition, the data analysis shines light on the fundamental physical properties of the excitonic transitions as function of layer number, in particular in the regime of few-layered nanosheets which are more difficult to study in micromechanically-exfoliated samples. We expect that both methodology and data analysis can be applied to other layered materials potentially giving access to identifying unifying properties across a range of $2 \mathrm{D}$ materials. 


\section{Methods}

\section{Sample Preparation}

TMD dispersions were prepared by probe sonicating the powder $\left(\mathrm{WS}_{2}\right.$ Sigma Aldrich C1254; $\mathrm{MoS}_{2}$ Sigma Aldrich 69860, MoSe 2 VWR 13112.14, WSe 2 VWR 13084.18) with an initial concentration $20 \mathrm{gL}^{-1}$ in an aqueous sodium cholate (SC) solution. The powder was immersed in $80 \mathrm{~mL}$ of aqueous surfactant solution $\left(\mathrm{CsC}_{\mathrm{SC}}=6 \mathrm{gL}^{-1}\right)$. The mixture was sonicated under icecooling in a $100 \mathrm{~mL}$ metal beaker by probe sonication using a solid flathead tip (Sonics VXC500 , i.e. $500 \mathrm{~W}$ ) for $1 \mathrm{~h}$ at $60 \%$ amplitude with a pulse of $6 \mathrm{~s}$ on and $2 \mathrm{~s}$ off. During the sonication, the sonic probe was placed $1.5 \mathrm{~cm}$ from the bottom of the beaker. The dispersion was centrifuged in $20 \mathrm{~mL}$ aliquots using $50 \mathrm{~mL}$ vials in a Hettich Mikro 220R centrifuge equipped with a fixed-angle rotor 1016 at 2,660 $g$ for $1.5 \mathrm{~h}$. The supernatant was discarded and the sediment collected in $80 \mathrm{~mL}$ of fresh surfactant $\left(\mathrm{CsC}=2 \mathrm{gL}^{-1}\right)$ and subjected to a second sonication using a solid flathead tip (Sonics VX-500) for $5 \mathrm{~h}$ at $60 \%$ amplitude with a pulse of $6 \mathrm{~s}$ on and $2 \mathrm{~s}$ off. From our experience, this two-step sonication procedure yields a higher concentration of exfoliated $\mathrm{WS}_{2}$ and removes impurities.

To select nanosheets by size, we used liquid cascade centrifugation with sequentially increasing rotation speeds $\left(2 \mathrm{~h}\right.$ each step, $\left.15^{\circ} \mathrm{C}\right)$. Centrifugation conditions are expressed as relative centrifugal field (RCF) in units of $10^{3} \times g$ (or $\mathrm{k} g$ ) with $g$ being the gravitational force. Two different rotors were used. For centrifugation at $<15,000 \mathrm{~g}$, the JA25.50 fixed angle rotor and $50 \mathrm{~mL}$ centrifuge tubes (VWR, order number 525-0402) were used filled with $20 \mathrm{~mL}$ of dispersion each. For centrifugation at $>15,000 \mathrm{~g}$, the JA25.15 rotor was used with $14 \mathrm{~mL}$ vials (Beckman Coulter, order number 331374), filled with $10 \mathrm{~mL}$ dispersion each.

The following procedure was applied as standard size selection of the primary cascade: Unexfoliated $\mathrm{WS}_{2}$ was removed by centrifugation at $100 \mathrm{~g}$. The supernatant was subjected to further centrifugation at $400 \mathrm{~g}$. The sediment was collected in fresh surfactant $(\mathrm{CsC}=0.1 \mathrm{gL}-1)$ at reduced volume $(3-8 \mathrm{~mL})$, while the supernatant was centrifuged at 1,000 $\mathrm{g}$. Again, the sediment was collected and the supernatant subjected to centrifugation at higher speeds. This procedure was repeated with the following RCF: 5,000 $\mathrm{g}, 10,000 \mathrm{~g}, 22,000 \mathrm{~g}, 74,000 \mathrm{~g}$. As sample nomenclature, the lower and upper boundary of the centrifugation are indicated.

In addition to this primary cascade, a subset of these size-selected $\mathrm{MoSe}_{2}$ and $\mathrm{WSe}_{2}$ dispersions were centrifuged overnight $(14 \mathrm{~h})$ at $1 / 5^{\text {th }}$ of the initial lower centrifugation boundary used to isolate the nanosheets initially. This procedure has previously shown to have the potential to 
change $\langle\mathrm{N}\rangle$ and $\langle\mathrm{L}\rangle$ independently ${ }^{78}$ especially when included in more complicated secondary cascades. ${ }^{18}$ Specifically, the $0.1-0.4 \mathrm{k} g$ fraction was centrifuged at $25 \mathrm{~g}$, the $0.4-1 \mathrm{k}$ $g$ fraction at $80 \mathrm{~g}$ and the $1.5 \mathrm{k} g$ fraction at $200 \mathrm{~g}$. In each case, the supernatant was subjected to analysis.

\section{Characterization}

Atomic force microscopy (AFM) was carried out on a Dimension ICON3 scanning probe microscope (Bruker AXS S.A.S.) with a $60 \mu \mathrm{m}^{2}$ scan head in tapping mode in air under ambient conditions using aluminium coated silicon cantilevers (OTESP-R3). Image sizes for small nanosheets were $5 \times 5 \mu \mathrm{m}^{2}$ with 1024 lines, and 10x10 $\mu \mathrm{m}^{2}$ with 1024 line per image for larger nanosheets, respectively. A drop of the dispersion $(10 \mu \mathrm{L})$ was drop-cast on pre-heated (150 $\left.{ }^{\circ} \mathrm{C}\right) \mathrm{Si} / \mathrm{SiO}_{2}$ wafer with an oxide layer of $300 \mathrm{~nm}$. The high concentration dispersions collected after LCC were diluted with water (to optical densities at C-exciton of 0.1-0.2) immediately prior to deposition to reduce the surfactant concentrations. After deposition, the wafer was rinsed with $\sim 5 \mathrm{~mL}$ of water and $\sim 5 \mathrm{~mL}$ of isopropanol. The apparent thickness was converted to number of layers using previously elaborated step-height analysis of liquid-exfoliated nanosheets.

Optical extinction was measured on a Varian Cary 6000i in quartz cuvettes with a pathlength of $0.4 \mathrm{~cm}$ in $0.5 \mathrm{~nm}$ increments and integration times of $0.1 \mathrm{~s} / \mathrm{nm}$. Samples were diluted to extinction values of $<0.5$ across the entire spectral region. To measure absorbance, the dispersions were placed in the center of an integrating sphere (external DRA-1800) and measured with integration times of $0.2 \mathrm{~s} / \mathrm{nm}$. To determine the extinction coefficients, nanosheet concentrations of a subset of dispersions were determined gravimetrically after filtration through alumina membranes, washing with $~ 500 \mathrm{~mL}$ of water, drying and weighing.

Photoluminescence was measured using a Horiba Scientific Fluorlog-3 spectrometer equipped with a Xe lamp $(450 \mathrm{~W})$ and a PPD-900 photomultiplier tube detector. Measurements were taken under ambient conditions with double monochromators for excitation and emission. Prior to the measurement, the samples were diluted to an optical density of 0.4 (with respect to $1 \mathrm{~cm}$ pathlength) at $430 \mathrm{~nm}$. The quartz cuvettes with $0.4 \times 1 \mathrm{~cm}$ dimensions were placed inside the spectrometer so that the excitation light passed through the $0.4 \mathrm{~cm}$ side of the cuvette. Emission was collected in a $90^{\circ}$ angle, i.e. after having passed through the $1 \mathrm{~cm}$ side. Bandwidths were $5 \mathrm{~nm}$, increments $1 \mathrm{~nm}$ and acquisition times $0.4 \mathrm{~s} / \mathrm{nm}$. To avoid artefacts from scattering of 
the nanomaterial dispersion, appropriate a $550 \mathrm{~nm}$ cut-off filters was placed on the emission side. The excitation was corrected for the light intensity.

\section{Theoretical methods}

Theoretical models of the band structure and exciton binding energies were performed based on the QEH model ${ }^{71}$ using the $a b$ initio package GPAW. ${ }^{76}$ Dielectric building blocks of monolayer $\mathrm{MoS}_{2}, \mathrm{MoSe}_{2}, \mathrm{WS}_{2}$ and $\mathrm{WSe}_{2}$ were retrieved from Ref. ${ }^{71}$. Band structures of the 2D material stacks studied were calculated using the G $\Delta \mathrm{W}-\mathrm{QEH}$ method. ${ }^{75}$

All structures were relaxed using the PBE exchange correlation functional until all forces on the atoms are smaller than $0.01 \mathrm{eV} / \AA$, and the lattice sizes in the $\mathrm{z}$-direction were chosen to be larger than $15 \AA$ to minimize the influence of periodic images. Ground-state wavefunctions were using PBE exchange correlation functional, with a plane-wave energy cutoff of $800 \mathrm{eV}$, 12x12x1 K-point grid, and a Fermi-Dirac distribution width of $0.01 \mathrm{eV}$. Quasi-particle band structure was computed using the $G_{0} W_{0}$ approximation to the PBE ground state wavefunction, with a total number of 128 bands to ensure convergence. To compute the band structure of $2 \mathrm{D}$ materials stacks, the dielectric screening matrix was first computed from the QEH model for a 2D material stack of $N$ layers to compute the correction to the eigenvalues $\Delta E_{n k}{ }^{i, s c r}$, such correction was further applied to the $G_{0} W_{0}$ band structure of monolayer to obtain the band structure of $N$ layers. The band structure was interpolated on the path $\Gamma-\mathrm{M}-\mathrm{K}-\Gamma$ to obtain direct bandgap $E_{g}(N)$ of $N$-layer stack.

The exciton binding energy $E_{\mathrm{b}}(N)$ was computed using the 2D Schrödinger equation, with the screened Coulomb potential $W$ obtained from the QEH model of $N$ layers. The theoretical Aexciton energy of N-layer 2D stack was then computed using $E_{\mathrm{A}}(N)=E_{\mathrm{g}}(N)-E_{\mathrm{b}}(N)$. 


\section{Figures}
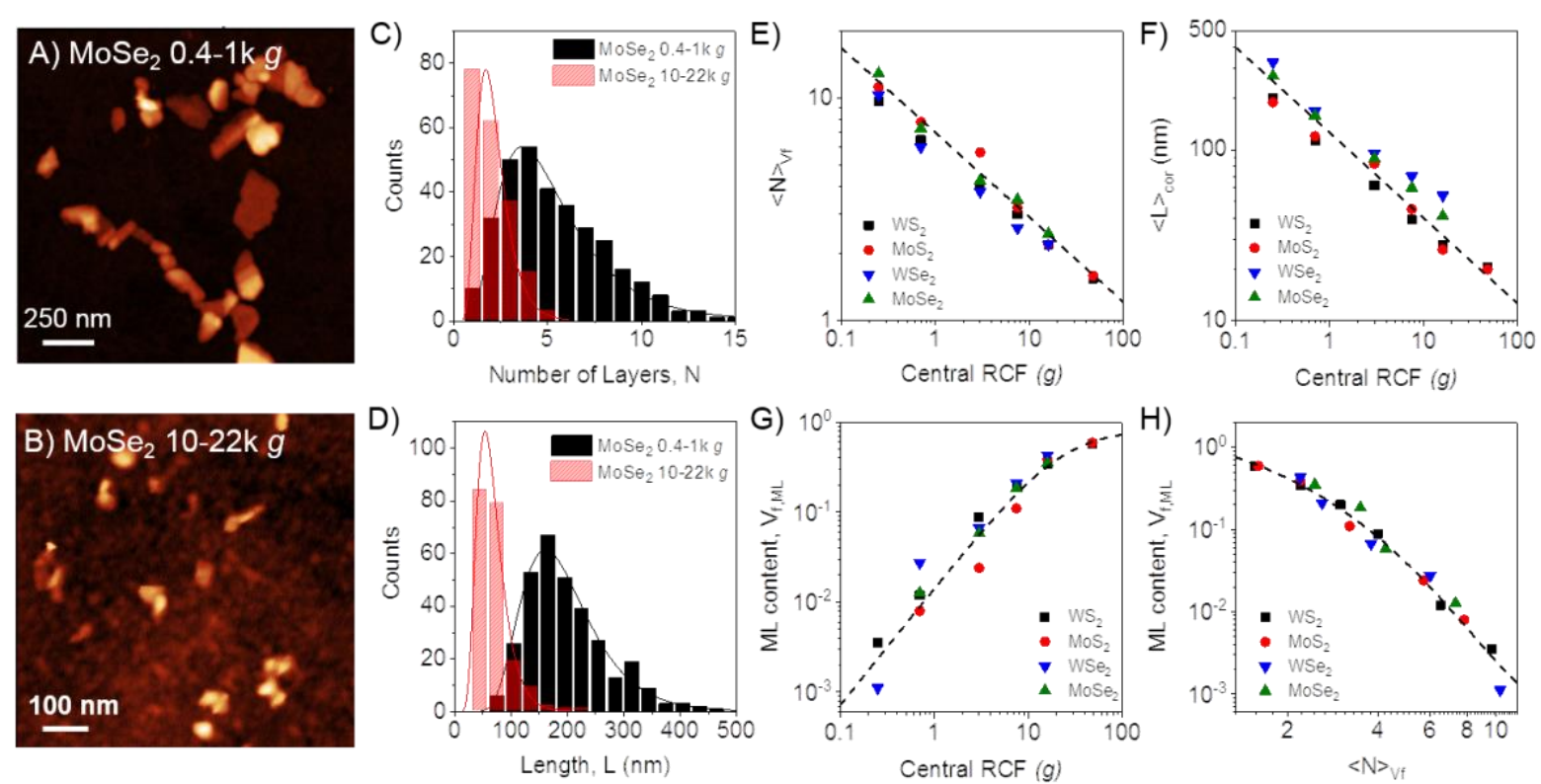

Figure 1: Quantification of average nanosheet dimensions by microscopy statistics. A, B)

Representative AFM images of large (Sample 0.4-1k g, A) and small (Sample 10-22k g, B) $\mathrm{MoSe}_{2}$ nanosheets. C,D) AFM number of layer (C) and length (D) histograms of large and small MoSe2, respectively. E-H) Comparison of mean nanosheet dimensions (according to AFM) after cascade centrifugation of different TMDs. E) Layer number, F) Mean nanosheet length, G) monolayer volume fraction as function of central centrifugal acceleration. $\mathrm{H}$ ) Relation between monolayer volume fraction and thickness. 

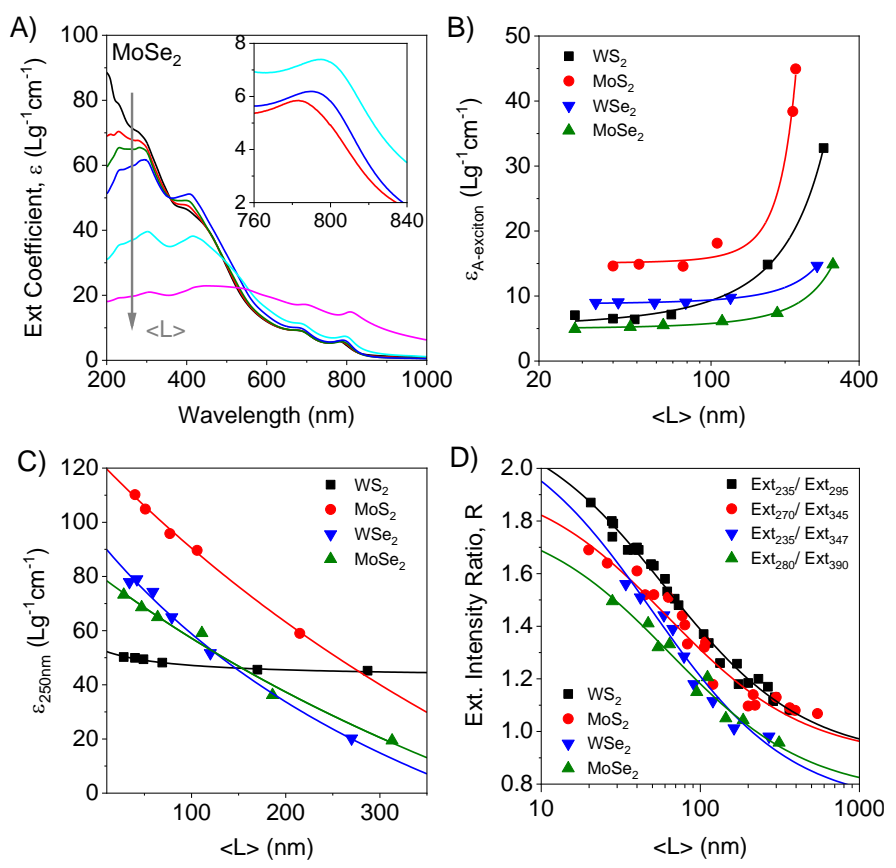

Figure 2: Impact of lateral nanosheet size on optical spectra. A) Extinction coefficient spectra of $\mathrm{MoSe}_{2}$ with different size/thickness distributions from ultraviolet to near-infrared spectral range. The arrow indicates where length changes influence the extinction coefficient significantly. Inset: A-exciton. The extinction spectra of the other TMDs are shown in the SI. B) Extinction coefficient at the A-exciton versus mean nanosheet length for four TMDs. C) Extinction coefficient at $250 \mathrm{~nm}$ versus mean nanosheet length for four TMDs. In this length regime, it depends linearly on $\langle\mathrm{L}\rangle$. D) Extinction intensity ratios as function of nanosheet length for different liquid-exfoliated TMDs. For $\mathrm{MoS}_{2}$ and $\mathrm{WS}_{2}$, previously published data is included. ${ }^{17,18}$ 

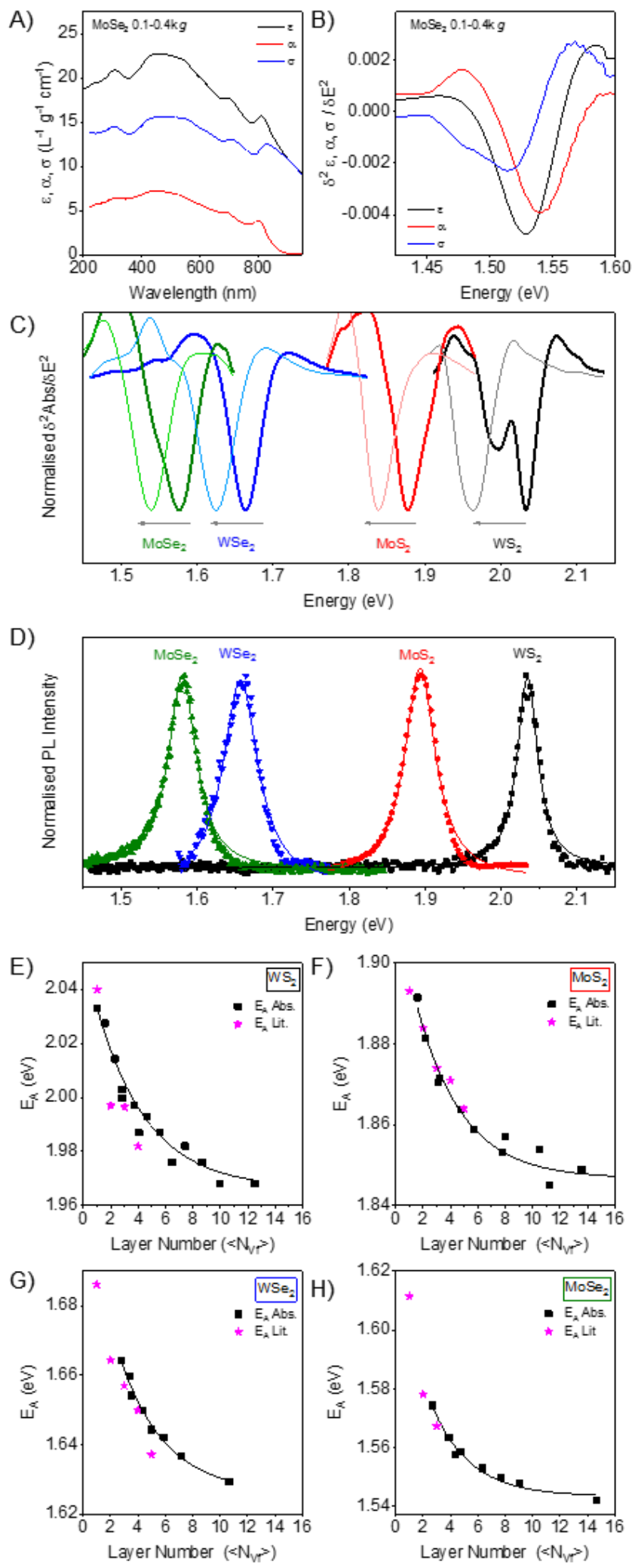

Figure 3: Impact of nanosheet layer number of optical properties. A) Extinction/absorbance/scattering spectra of a fraction of large $\mathrm{MoSe}_{2}$ nanosheets. The contribution from scattering is non-negligible and can even be larger than the absorbance. B) Second derivative of the A-exciton of the sample in A). Scattering in the non-resonant is red- 
shifted. Therefore, when scattering is non-negligible, extinction peak positions are red-shifted compared to absorbance. C) A-exciton second derivative of TMD absorbance spectra of dispersions containing thin (darker line) and thick (lighter line) nanosheets. The arrows indicate the thickness dependent shift, D) Photoluminescence spectra of monolayer-rich samples (measured in dispersion with an excitation wavelength of $435 \mathrm{~nm}$ ), E-H) Plot of A-exciton energy from absorbance as function of layer number of the TMDs compared to data from literature adjusted by a constant offset in each case, E) $\left.\left.\mathrm{WS}_{2},{ }^{20} \mathrm{~F}\right) \mathrm{MoS}_{2},{ }^{12} \mathrm{G}\right) \mathrm{WSe}_{2},{ }^{20} \mathrm{H}$ ) $\operatorname{MoSe}_{2} \cdot{ }^{19}$ For $\mathrm{WS}_{2}$ and $\mathrm{MoS}_{2}$, previously published data on LPE nanosheets is also included. ${ }^{17}$, ${ }^{18}$ Lines are exponential fits that are used to extract the A-exciton energy of the bulk, the monolayer and the rate at which the monolayer approaches the bulk values. 

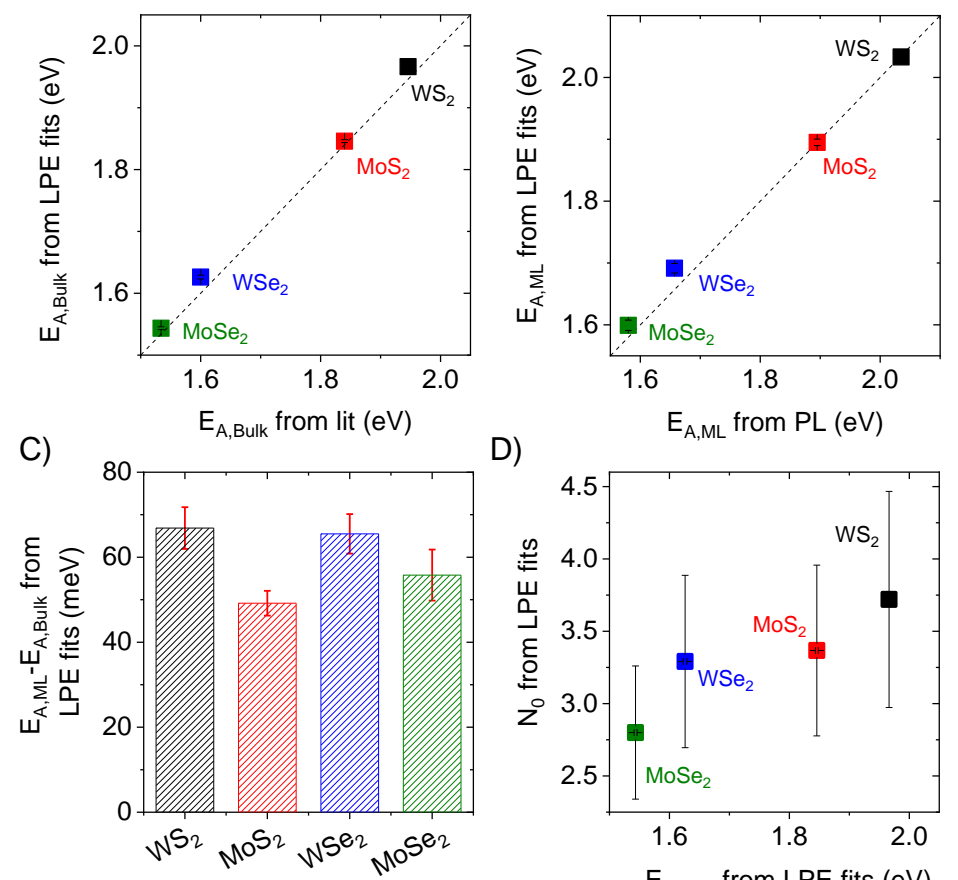

D)
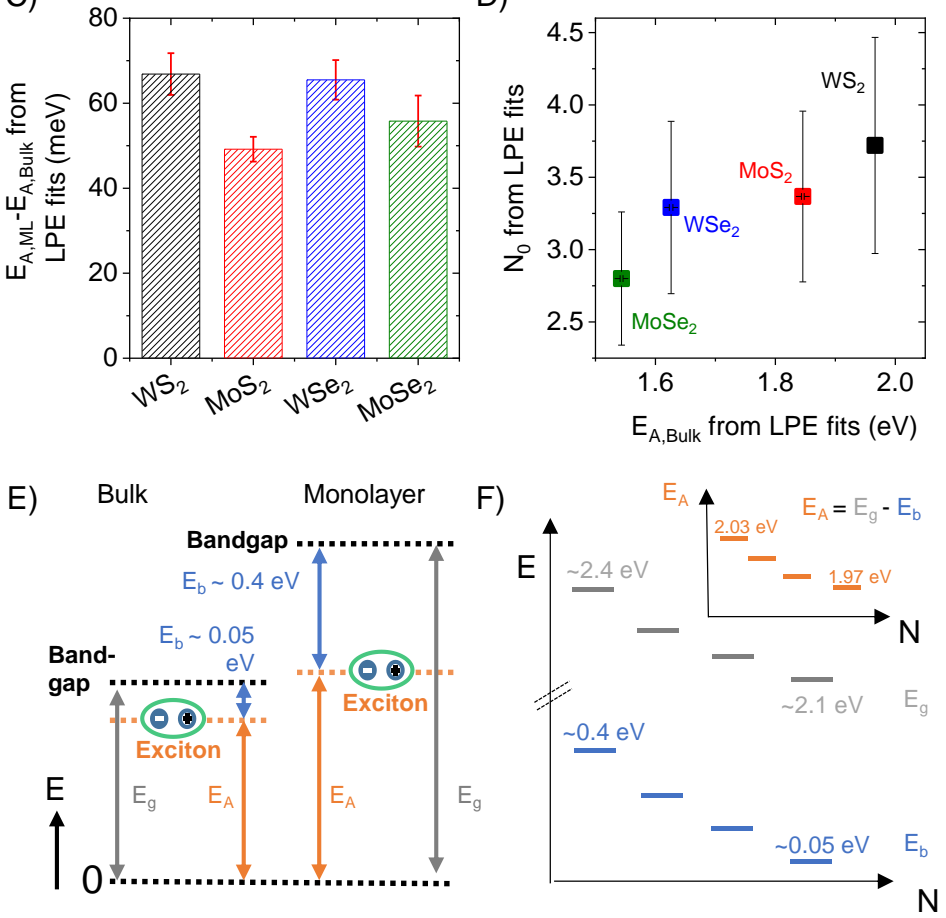

Figure 4: Results of fitting the exponential A-exciton - layer number dependence. A) Plot of the bulk exciton energy extracted from the fit of the LPE samples versus literature data. ${ }^{52}$ The line represents $y=x$. B) Plot of bulk monolayer energy extracted from the fit of the LPE samples versus the position of the PL lines presented in figure 3D. C) Plot of the difference in monolayer and bulk exciton energies for the different TMDs. D) Decay constant representing rate at which the monolayer A-exciton approaches the bulk value as function of bulk exciton energy. E-F) Schematic representation of changes in the band gap at the $\mathrm{K}$ point and A-exciton binding energies as function of layer number. The indicated values are taken from the literature. 15, 58 Both the band gap at the K-point and the A-exciton binding energies decrease by 0.3-0.35 $\mathrm{eV}$ as the layer number is increased from monolayer to bulk. As a result, the optical gap (i.e. the measured A-exciton binding energy) only weakly depends on layer number, but in a welldefined way (see figure 3). 

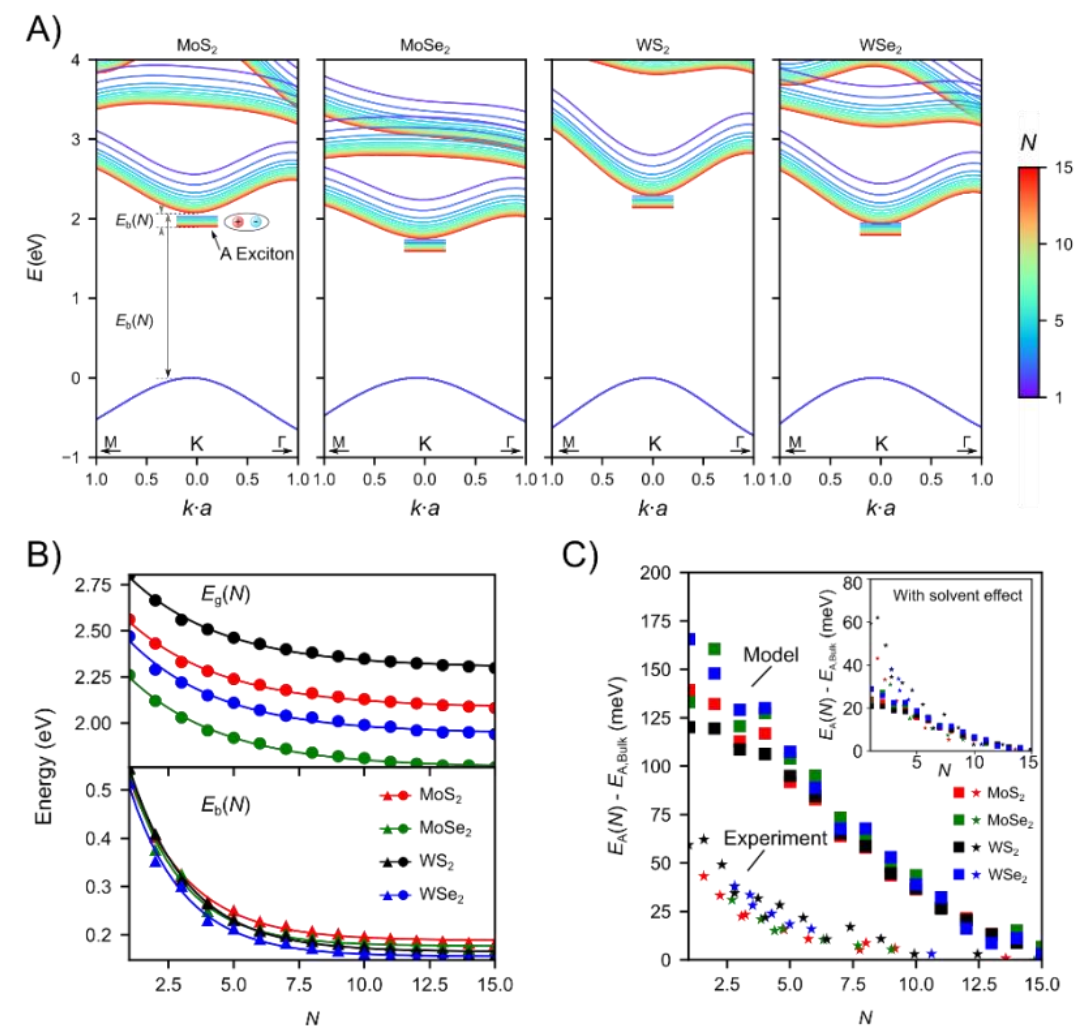

C)
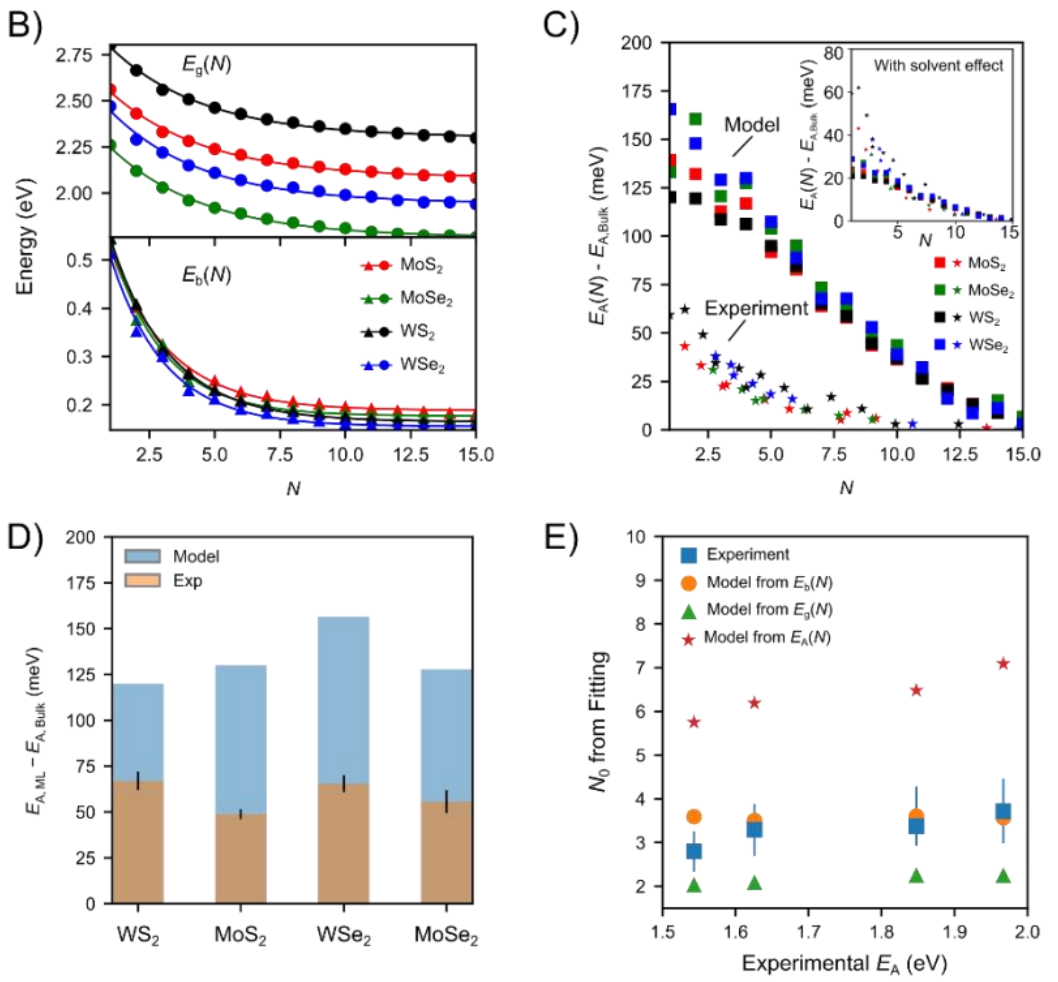

Figure 5: Theoretical investigation of the evolution of A exciton peak using the QEH model. A) Evolution of the band structure around the K point for several 2D semiconductors with the color map indicating the number of 2D layers. The valence band (VB) of all materials are set to zero at the Fermi level. The conduction band (CB) shifts to lower energy with increasing number of $\mathrm{N}$ due to the increased dielectric screening. The positions of the A exciton are schematically illustrated by using horizontal lines below the $\mathrm{CB}$ valley at the $\mathrm{K}$ point. B) Calculated evolution of the band gap $E_{g}(N)$ (circles) and the excitonic binding energy $E_{b}(N)$ (triangles) as a function of the layer number $\mathrm{N}$ which indicates a similar trend of decay due to the dielectric screening. Solid lines represent the fitting results using exponential decay model. C) Comparison between the change of the energy of the A exciton, $E_{A}(N)-E_{A, B u l k}$, obtained from experimental data (stars) and the QEH model (squares), showing much small degree of change compared with $\operatorname{Eg}(\mathrm{N})$ and $\operatorname{Eb}(\mathrm{N})$. Solvent effects are considered in the inset 
where the dielectric constant of the solvent $(\sim 1.37)$ is included. D) Comparison between the values of $\mathrm{E}_{\mathrm{A}, \mathrm{ML}}-\mathrm{E}_{\mathrm{A}, \mathrm{Bulk}}$ from experimental data and QEH model. E) Comparison between the $N_{0}$ values obtained from experimental data and fitting of $E_{g}(N), E_{b}(N)$ and $E_{A}(B)$ data from QEH model. The values of $\mathrm{N}_{0}$ from simulation using $\mathrm{E}_{\mathrm{b}}$ agree very close the with experimental observations.

Table 1: Fit parameters found by fitting extinction ratio, $\mathrm{R}$, versus mean nanosheet length (figure 2D) to eq 3, for each of the four TMDs. Once these parameters are known, equation 3 can be rearrange to give $\langle\mathrm{L}\rangle$ as a function of $\mathrm{R}$.

\begin{tabular}{|l|l|l|l|l|l|l|}
\hline & $\lambda_{1}(\mathrm{~nm})$ & $\lambda_{2}(\mathrm{~nm})$ & $\mathrm{A}_{1}\left(\mathrm{~nm}^{-1}\right)$ & $\mathrm{B}_{1}$ & $\mathrm{~A}_{2}\left(\mathrm{~nm}^{-1}\right)$ & $\mathrm{B}_{2}$ \\
\hline $\mathrm{MoS}_{2}$ & 270 & 345 & 0.0144 & 1.97 & 0.0160 & 1 \\
\hline $\mathrm{MoSe}_{2}$ & 280 & 390 & 0.0117 & 1.83 & 0.0154 & 1 \\
\hline $\mathrm{WS}_{2}$ & 235 & 295 & 0.0159 & 2.20 & 0.0166 & 1 \\
\hline WSe $_{2}$ & 235 & 347 & 0.0143 & 2.20 & 0.0200 & 1 \\
\hline
\end{tabular}

Table 2: Mean nanosheet thickness, monolayer volume fraction, centre of A-exciton peak in second derivative of highly monolayer-enriched absorbance spectrum as well as centre and FWHM of PL spectra found by Lorentzian fitting.

\begin{tabular}{|l|l|l|l|l|l|}
\hline & $\langle N\rangle_{V f}$ & ML Vf & Abs, E0 (eV) & PL, E0 (eV) & PL, $\Delta \mathrm{E}(\mathrm{meV})$ \\
\hline $\mathrm{MoS}_{2}$ & 1.6 & $60 \%$ & 1.877 & 1.891 & 48 \\
\hline $\mathrm{MoSe}_{2}$ & 2.5 & $27 \%$ & 1.577 & 1.573 & 45 \\
\hline $\mathrm{WS}_{2}$ & 1.6 & $60 \%$ & $\begin{array}{l}2.034 \quad(\mathrm{ML} \\
\text { component })\end{array}$ & 2.032 & 33 \\
\hline $\mathrm{WSe}_{2}$ & 2.3 & $31 \%$ & 1.664 & 1.657 & 51 \\
\hline
\end{tabular}


Table 3: Exponential decay fit parameters for A exciton position versus mean nanosheet thickness (layer number) data (figure $3 \mathrm{E}-\mathrm{H}$ ).

\begin{tabular}{|l|l|l|l|}
\hline & EA,ML $(\mathrm{eV})$ & EA,Bulk $^{(\mathrm{eV})}$ & $\mathrm{N}_{0}$ \\
\hline $\mathrm{MoS}_{2}$ & 1.895 & 1.846 & 3.37 \\
\hline $\mathrm{MoSe}_{2}$ & 1.599 & 1.544 & 2.80 \\
\hline $\mathrm{WS}_{2}$ & 2.033 & 1.966 & 3.72 \\
\hline $\mathrm{WSe}_{2}$ & 1.692 & 1.626 & 3.29 \\
\hline
\end{tabular}

\section{Acknowledgement}

The research leading to these results has received funding from the European Union's Horizon 2020 under grant agreement $n^{\circ} 785219$ Graphene Flagship and the European research Council Advanced Grant FUTURE-PRINT. C.B. acknowledges the German research foundation DFG under Emmy-Noether grant BA4856/2-1. A.C. acknowledges the German research foundation DFG under Emmy-Noether grant CH 1672/1-1. E.J.G.S. acknowledges the use of computational resources from the UK Materials and Molecular Modelling Hub for access to THOMAS supercluster, which is partially funded by EPSRC (EP/P020194/1). The Queen's Fellow Award through the grant number M8407MPH, and the Department for the Economy (USI 097) are also acknowledged. We thank Jana Zaumseil for access to the infrastructure at the Chair of Applied Physical Chemistry, Heidelberg.

\section{Additional information}

Supplementary information is available.

\section{Competing financial interests}

The authors declare no competing financial interests. 


\section{References}

1. Chhowalla, M.; Shin, H. S.; Eda, G.; Li, L. J.; Loh, K. P.; Zhang, H., The Chemistry of TwoDimensional Layered Transition Metal Dichalcogenide Nanosheets. Nature Chem. 2013, 5, 263-275. 2. $\quad$ Wang, Q. H.; Kalantar-Zadeh, K.; Kis, A.; Coleman, J. N.; Strano, M. S., Electronics and Optoelectronics of Two-Dimensional Transition Metal Dichalcogenides. Nat. Nanotechnol. 2012, 7 , 699-712.

3. Jariwala, D.; Sangwan, V. K.; Lauhon, L. J.; Marks, T. J.; Hersam, M. C., Emerging Device Applications for Semiconducting Two-Dimensional Transition Metal Dichalcogenides. ACS Nano 2014, 8, 1102-1120.

4. Mak, K. F.; Shan, J., Photonics and Optoelectronics of 2d Semiconductor Transition Metal Dichalcogenides. Nat Photon 2016, 10, 216-226.

5. Buscema, M.; Island, J. O.; Groenendijk, D. J.; Blanter, S. I.; Steele, G. A.; van der Zant, H. S. J.; Castellanos-Gomez, A., Photocurrent Generation with Two-Dimensional Van Der Waals Semiconductors. Chem. Soc. Rev. 2015, 44, 3691-3718.

6. Voiry, D.; Yang, J.; Chhowalla, M., Recent Strategies for Improving the Catalytic Activity of 2d Tmd Nanosheets toward the Hydrogen Evolution Reaction. Adv. Mater. 2016, 28, 6197-6206.

7. $\quad$ Low, T.; Chaves, A.; Caldwell, J. D.; Kumar, A.; Fang, N. X.; Avouris, P.; Heinz, T. F.; Guinea, F.; Martin-Moreno, L.; Koppens, F., Polaritons in Layered Two-Dimensional Materials. Nat. Mater. 2017, 16, 182-194.

8. $\quad$ Li, B. L.; Wang, J.; Zou, H. L.; Garaj, S.; Lim, C. T.; Xie, J.; Li, N. B.; Leong, D. T., LowDimensional Transition Metal Dichalcogenide Nanostructures Based Sensors. Adv. Funct. Mater. 2016, 26, 7034-7056.

9. $\quad$ Tao, H.; Gao, Y.; Talreja, N.; Guo, F.; Texter, J.; Sun, Z.; Yan, C., Two-Dimensional Nanosheets for Electrocatalysis in Energy Generation and Conversion. J. Mater. Chem. C. 2017, 16. 10. Pumera, M.; Sofer, Z.; Ambrosi, A., Layered Transition Metal Dichalcogenides for Electrochemical Energy Generation and Storage. J. Mater. Chem. C. 2014, 2, 8981-8987.

11. Mendoza-Sánchez, B.; Gogotsi, Y., Synthesis of Two-Dimensional Materials for Capacitive Energy Storage. Adv. Mater. 2016, 28, 6104-6135.

12. Mak, K. F.; Lee, C.; Hone, J.; Shan, J.; Heinz, T. F., Atomically Thin Mos2: A New DirectGap Semiconductor. Phys. Rev. Lett. 2010, 105.

13. Ross, J. S.; Klement, P.; Jones, A. M.; Ghimire, N. J.; Yan, J. Q.; Mandrus, D. G.; Taniguchi, T.; Watanabe, K.; Kitamura, K.; Yao, W.; Cobden, D. H.; Xu, X. D., Electrically Tunable Excitonic Light-Emitting Diodes Based on Monolayer Wse2 P-N Junctions. Nat. Nanotechnol. 2014, 9, 268 272.

14. Amani, M.; Lien, D. H.; Kiriya, D.; Xiao, J.; Azcatl, A.; Noh, J.; Madhvapathy, S. R.; Addou, R.; Santosh, K. C.; Dubey, M.; Cho, K.; Wallace, R. M.; Lee, S. C.; He, J. H.; Ager, J. W.; Zhang, X.; Yablonovitch, E.; Javey, A., Near-Unity Photoluminescence Quantum Yield in Mos2. Science 2015, 350, 1065-1068.

15. Wang, G.; Chernikov, A.; Glazov, M. M.; Heinz, T. F.; Marie, X.; Amand, T.; Urbaszek, B., Colloquium: Excitons in Atomically Thin Transition Metal Dichalcogenides. Reviews of Modern Physics 2018, 90.

16. Niu, Y.; Gonzalez-Abad, S.; Frisenda, R.; Marauhn, P.; Drüppel, M.; Gant, P.; Schmidt, R.; Taghavi, N.; Barcons, D.; Molina-Mendoza, A.; de Vasconcellos, S.; Bratschitsch, R.; Perez De Lara, D.; Rohlfing, M.; Castellanos-Gomez, A., Thickness-Dependent Differential Reflectance Spectra of Monolayer and Few-Layer Mos2, Mose2, Ws2 and Wse2. Nanomaterials 2018, 8, 725.

17. Backes, C.; Smith, R. J.; McEvoy, N.; Berner, N. C.; McCloskey, D.; Nerl, H. C.; O’Neill, A.; King, P. J.; Higgins, T.; Hanlon, D.; Scheuschner, N.; Maultzsch, J.; Houben, L.; Duesberg, G. S.; Donegan, J. F.; Nicolosi, V.; Coleman, J. N., Edge and Confinement Effects Allow in Situ Measurement of Size and Thickness of Liquid-Exfoliated Nanosheets. Nature Commun. 2014, 5, 4576.

18. Backes, C.; Szydlowska, B. M.; Harvey, A.; Yuan, S.; Vega-Mayoral, V.; Davies, B. R.; Zhao, P. L.; Hanlon, D.; Santos, E. J.; Katsnelson, M. I.; Blau, W. J.; Gadermaier, C.; Coleman, J. N., 
Production of Highly Monolayer Enriched Dispersions of Liquid-Exfoliated Nanosheets by Liquid Cascade Centrifugation. ACS Nano 2016, 10, 1589-601.

19. Tonndorf, P.; Schmidt, R.; Böttger, P.; Zhang, X.; Börner, J.; Liebig, A.; Albrecht, M.; Kloc, C.; Gordan, O.; Zahn, D. R. T.; Michaelis de Vasconcellos, S.; Bratschitsch, R., Photoluminescence Emission and Raman Response of Monolayer Mos2, Mose2, and Wse2. Optics Express 2013, 21, 4908-4916.

20. Zhao, W. J.; Ghorannevis, Z.; Chu, L. Q.; Toh, M. L.; Kloc, C.; Tan, P. H.; Eda, G., Evolution of Electronic Structure in Atomically Thin Sheets of Ws2 and Wse2. ACS Nano 2013, 7, 791-797.

21. Coleman, J. N.; Lotya, M.; O'Neill, A.; Bergin, S. D.; King, P. J.; Khan, U.; Young, K.; Gaucher, A.; De, S.; Smith, R. J.; Shvets, I. V.; Arora, S. K.; Stanton, G.; Kim, H. Y.; Lee, K.; Kim, G. T.; Duesberg, G. S.; Hallam, T.; Boland, J. J.; Wang, J. J.; Donegan, J. F.; Grunlan, J. C.; Moriarty, G.; Shmeliov, A.; Nicholls, R. J.; Perkins, J. M.; Grieveson, E. M.; Theuwissen, K.; McComb, D. W.; Nellist, P. D.; Nicolosi, V., Two-Dimensional Nanosheets Produced by Liquid Exfoliation of Layered Materials. Science 2011, 331, 568-571.

22. Paton, K. R.; Varrla, E.; Backes, C.; Smith, R. J.; Khan, U.; O'Neill, A.; Boland, C.; Lotya, M.; Istrate, O. M.; King, P.; Higgins, T.; Barwich, S.; May, P.; Puczkarski, P.; Ahmed, I.; Moebius, M.; Pettersson, H.; Long, E.; Coelho, J.; O'Brien, S. E.; McGuire, E. K.; Sanchez, B. M.; Duesberg, G. S.; McEvoy, N.; Pennycook, T. J.; Downing, C.; Crossley, A.; Nicolosi, V.; Coleman, J. N., Scalable Production of Large Quantities of Defect-Free Few-Layer Graphene by Shear Exfoliation in Liquids. Nature Materials 2014, 13, 624-630.

23. Del Rio Castillo, A. E.; Pellegrini, V.; Ansaldo, A.; Ricciardella, F.; Sun, H.; Marasco, L.; Buha, J.; Dang, Z.; Gagliani, L.; Lago, E.; Curreli, N.; Gentiluomo, S.; Palazon, F.; Prato, M.; Oropesa-Nuñez, R.; Toth, P. S.; Mantero, E.; Crugliano, M.; Gamucci, A.; Tomadin, A.; Polini, M.; Bonaccorso, F., High-Yield Production of 2d Crystals by Wet-Jet Milling. Materials Horizons 2018, $5,890-904$.

24. Harvey, A.; He, X. Y.; Godwin, I. J.; Backes, C.; McAteer, D.; Berner, N. C.; McEvoy, N.; Ferguson, A.; Shmeliov, A.; Lyons, M. E. G.; Nicolosi, V.; Duesberg, G. S.; Donegan, J. F.; Coleman, J. N., Production of $\mathrm{Ni}(\mathrm{Oh})(2)$ Nanosheets by Liquid Phase Exfoliation: From Optical Properties to Electrochemical Applications. J. Mater. Chem. C. 2016, 4, 11046-11059.

25. Coleman, J. N., Liquid Exfoliation of Defect-Free Graphene. Acc. Chem. Res. 2013, 46, 14-

22.

26. Khan, U.; Porwal, H.; O'Neill, A.; Nawaz, K.; May, P.; Coleman, J. N., Solvent-Exfoliated Graphene at Extremely High Concentration. Langmuir 2011, 27, 9077-9082.

27. Backes, C.; Campi, D.; Szydlowska, B. M.; Synnatschke, K.; Ojala, E.; Rashvand, F.; Harvey, A.; Griffin, A.; Sofer, Z.; Marzari, N.; Coleman, J. N.; O’Regan, D. D., Equipartition of Energy Defines the Size-Thickness Relationship in Liquid-Exfoliated Nanosheets. ACS Nano 2019, 13, 70507061.

28. Yi, M.; Shen, Z. G., Kitchen Blender for Producing High-Quality Few-Layer Graphene. Carbon 2014, 78, 622-626.

29. Hernandez, Y.; Nicolosi, V.; Lotya, M.; Blighe, F. M.; Sun, Z. Y.; De, S.; McGovern, I. T.; Holland, B.; Byrne, M.; Gun'ko, Y. K.; Boland, J. J.; Niraj, P.; Duesberg, G.; Krishnamurthy, S.; Goodhue, R.; Hutchison, J.; Scardaci, V.; Ferrari, A. C.; Coleman, J. N., High-Yield Production of Graphene by Liquid-Phase Exfoliation of Graphite. Nat. Nanotechnol. 2008, 3, 563-568.

30. Liu, L. H.; Zorn, G.; Castner, D. G.; Solanki, R.; Lerner, M. M.; Yan, M. D., A Simple and Scalable Route to Wafer-Size Patterned Graphene. J. Mater. Chem. 2010, 20, 5041-5046.

31. Du, W. C.; Jiang, X. Q.; Zhu, L. H., From Graphite to Graphene: Direct Liquid-Phase Exfoliation of Graphite to Produce Single- and Few-Layered Pristine Graphene. J. Mater. Chem. C. 2013, 1, 10592-10606.

32. Zhi, C. Y.; Bando, Y.; Tang, C. C.; Kuwahara, H.; Golberg, D., Large-Scale Fabrication of Boron Nitride Nanosheets and Their Utilization in Polymeric Composites with Improved Thermal and Mechanical Properties. Adv. Mater. 2009, 21, 2889-+.

33. Zhu, J.; Kang, J.; Kang, J.; Jariwala, D.; Wood, J. D.; Seo, J.-W. T.; Chen, K.-S.; Marks, T. J.; Hersam, M. C., Solution-Processed Dielectrics Based on Thickness-Sorted Two-Dimensional Hexagonal Boron Nitride Nanosheets. Nano Lett. 2015, 15, 7029-7036. 
34. Bang, G. S.; Nam, K. W.; Kim, J. Y.; Shin, J.; Choi, J. W.; Choi, S. Y., Effective LiquidPhase Exfoliation and Sodium Ion Battery Application of Mos2 Nanosheets. ACS Appl. Mater. Interfaces 2014, 6, 7084-7089.

35. Vega-Mayoral, V.; Tian, R. Y.; Kelly, A. G.; Griffin, A.; Harvey, A.; Borrelli, M.; Nisi, K.; Backes, C.; Coleman, J. N., Solvent Exfoliation Stabilizes Tis2 Nanosheets against Oxidation, Facilitating Lithium Storage Applications. Nanoscale 2019, 11, 6206-6216.

36. Kang, J.; Sangwan, V. K.; Wood, J. D.; Liu, X.; Balla, I.; Lam, D.; Hersam, M. C., Layer-byLayer Sorting of Rhenium Disulfide Via High-Density Isopycnic Density Gradient Ultracentrifugation. Nano Lett. 2016, 16, 7216-7223.

37. Harvey, A.; Backes, C.; Gholamvand, Z.; Hanlon, D.; McAteer, D.; Nerl, H. C.; McGuire, E.; Seral-Ascaso, A.; Ramasse, Q. M.; McEvoy, N.; Winters, S.; Berner, N. C.; McCloskey, D.; Donegan, J. F.; Duesberg, G. S.; Nicolosi, V.; Coleman, J. N., Preparation of Gallium Sulfide Nanosheets by Liquid Exfoliation and Their Application as Hydrogen Evolution Catalysts. Chem. Mater. 2015, 27, 3483-3493.

38. Petroni, E.; Lago, E.; Bellani, S.; Boukhvalov, D. W.; Politano, A.; Gürbulak, B.; Duman, S.; Prato, M.; Gentiluomo, S.; Oropesa-Nuñez, R.; Panda, J.-K.; Toth, P. S.; Del Rio Castillo, A. E.; Pellegrini, V.; Bonaccorso, F., Liquid-Phase Exfoliated Indium-Selenide Flakes and Their Application in Hydrogen Evolution Reaction. Small 2018, 14, 1800749.

39. Brent, J. R.; Lewis, D. J.; Lorenz, T.; Lewis, E. A.; Savjani, N.; Haigh, S. J.; Seifert, G.; Derby, B.; O'Brien, P., Tin(Ii) Sulfide (Sns) Nanosheets by Liquid-Phase Exfoliation of Herzenbergite: Iv-Vi Main Group Two-Dimensional Atomic Crystals. J. Am. Chem. Soc. 2015, 137, 12689-12696.

40. $\quad$ Zhang, P.; Zhao, F.; Long, P.; Wang, Y.; Yue, Y.; Liu, X.; Feng, Y.; Li, R.; Hu, W.; Li, Y.; Feng, W., Sonication-Assisted Liquid-Phase Exfoliated A-Gete: A Two-Dimensional Material with High Fe3+ Sensitivity. Nanoscale 2018, 10, 15989-15997.

41. Paton, K. R.; Varrla, E.; Backes, C.; Smith, R. J.; Khan, U.; O'Neill, A.; Boland, C.; Lotya, M.; Istrate, O. M.; King, P.; Higgins, T.; Barwich, S.; May, P.; Puczkarski, P.; Ahmed, I.; Moebius, M.; Pettersson, H.; Long, E.; Coelho, J.; O'Brien, S. E.; McGuire, E. K.; Sanchez, B. M.; Duesberg, G. S.; McEvoy, N.; Pennycook, T. J.; Downing, C.; Crossley, A.; Nicolosi, V.; Coleman, J. N., Scalable Production of Large Quantities of Defect-Free Few-Layer Graphene by Shear Exfoliation in Liquids. Nat. Mater. 2014, 13, 624-30.

42. O'Connell, M. J.; Bachilo, S. M.; Huffman, C. B.; Moore, V. C.; Strano, M. S.; Haroz, E. H.; Rialon, K. L.; Boul, P. J.; Noon, W. H.; Kittrell, C.; Ma, J. P.; Hauge, R. H.; Weisman, R. B.; Smalley, R. E., Band Gap Fluorescence from Individual Single-Walled Carbon Nanotubes. Science 2002, 297, 593-596.

43. $\quad$ Backes, C.; Paton, K. R.; Hanlon, D.; Yuan, S.; Katsnelson, M. I.; Houston, J.; Smith, R. J.; McCloskey, D.; Donegan, J. F.; Coleman, J. N., Spectroscopic Metrics Allow in Situ Measurement of Mean Size and Thickness of Liquid-Exfoliated Few-Layer Graphene Nanosheets. Nanoscale 2016, 8, 4311-4323.

44. Hanlon, D.; Backes, C.; Doherty, E.; Cucinotta, C. S.; Berner, N. C.; Boland, C.; Lee, K.; Harvey, A.; Lynch, P.; Gholamvand, Z.; Zhang, S. F.; Wang, K. P.; Moynihan, G.; Pokle, A.; Ramasse, Q. M.; McEvoy, N.; Blau, W. J.; Wang, J.; Abellan, G.; Hauke, F.; Hirsch, A.; Sanvito, S.; O'Regan, D. D.; Duesberg, G. S.; Nicolosi, V.; Coleman, J. N., Liquid Exfoliation of SolventStabilized Few-Layer Black Phosphorus for Applications Beyond Electronics. Nature Commun. 2015, 6.

45. Singh, M.; Della Gaspera, E.; Ahmed, T.; Walia, S.; Ramanathan, R.; van Embden, J.; Mayes, E.; Bansal, V., Soft Exfoliation of 2d Sno with Size-Dependent Optical Properties. 2D Mater. 2017, 4. 46. Woomer, A. H.; Farnsworth, T. W.; Hu, J.; Wells, R. A.; Donley, C. L.; Warren, S. C., Phosphorene: Synthesis, Scale-up, and Quantitative Optical Spectroscopy. ACS Nano 2015, 9, 88698884 .

47. Wilson, J. A.; Yoffe, A. D., Transition Metal Dichalcogenides Discussion and Interpretation of Observed Optical, Electrical and Structural Properties. Advances in Physics 1969, 18, 193-\&.

48. van de Hulst, H. C., Light Scattering by Small Particles. Courier Corporation: 1981. 
49. Yadgarov, L.; Choi, C. L.; Sedova, A.; Cohen, A.; Rosentsveig, R.; Bar-Elli, O.; Oron, D.; Dai, H. J.; Tenne, R., Dependence of the Absorption and Optical Surface Plasmon Scattering of Mos2 Nanoparticles on Aspect Ratio, Size, and Media. ACS Nano 2014, 8, 3575-3583.

50. $\quad$ Harvey, A.; Backes, C.; Boland, J. B.; He, X.; Griffin, A.; Szydlowska, B.; Gabbett, C.; Donegan, J. F.; Coleman, J. N., Non-Resonant Light Scattering in Dispersions of 2d Nanosheets. Nature Commun. 2018, 9, 4553.

51. Mak, K. F.; He, K. L.; Lee, C.; Lee, G. H.; Hone, J.; Heinz, T. F.; Shan, J., Tightly Bound Trions in Monolayer Mos2. Nat. Mater. 2013, 12, 207-211.

52. Li, Y. L.; Chernikov, A.; Zhang, X.; Rigosi, A.; Hill, H. M.; van der Zande, A. M.; Chenet, D. A.; Shih, E. M.; Hone, J.; Heinz, T. F., Measurement of the Optical Dielectric Function of Monolayer Transition-Metal Dichalcogenides: Mos2, Mose2, Ws2, and Wse2. Phys. Rev. B 2014, 90.

53. Selig, M.; Berghauser, G.; Raja, A.; Nagler, P.; Schuller, C.; Heinz, T. F.; Korn, T.; Chernikov, A.; Malic, E.; Knorr, A., Excitonic Linewidth and Coherence Lifetime in Monolayer Transition Metal Dichalcogenides. Nature Commun. 2016, 7.

54. Kormanyos, A.; Burkard, G.; Gmitra, M.; Fabian, J.; Zolyomi, V.; Drummond, N. D.; Fal'ko, V., K.P Theory for Two-Dimensional Transition Metal Dichalcogenide Semiconductors. 2D Mater. 2015, 2.

55. Jin, Z. H.; Li, X. D.; Mullen, J. T.; Kim, K. W., Intrinsic Transport Properties of Electrons and Holes in Monolayer Transition-Metal Dichalcogenides. Phys. Rev. B 2014, 90.

56. Beal, A. R.; Hughes, H. P., Kramers-Kronig Analysis of the Reflectivity Spectra of 2h-Mos2, 2h-Mose2and 2h-Mote2. Journal of Physics C: Solid State Physics 1979, 12, 881-890.

57. Beal, A. R.; Liang, W. Y.; Hughes, H. P., Kramers-Kronig Analysis of the Reflectivity Spectra of 3r-Ws2and 2h-Wse2. Journal of Physics C: Solid State Physics 1976, 9, 2449-2457. 58. Evans, B. L.; Young, P. A., Exciton Spectra in Thin Crystals: The Diamagnetic Effect. Proceedings of the Physical Society 1967, 91, 475-482.

59. Beal, A. R.; Liang, W. Y., Excitons in 2h-Wse2 and 3r-Ws2. J. Phys. C 1976, 9, 2459-2466.

60. Ugeda, M. M.; Bradley, A. J.; Shi, S. F.; da Jornada, F. H.; Zhang, Y.; Qiu, D. Y.; Ruan, W.; Mo, S. K.; Hussain, Z.; Shen, Z. X.; Wang, F.; Louie, S. G.; Crommie, M. F., Giant Bandgap

Renormalization and Excitonic Effects in a Monolayer Transition Metal Dichalcogenide Semiconductor. Nat. Mater. 2014, 13, 1091-1095.

61. Chernikov, A.; Berkelbach, T. C.; Hill, H. M.; Rigosi, A.; Li, Y. L.; Aslan, O. B.; Reichman, D. R.; Hybertsen, M. S.; Heinz, T. F., Exciton Binding Energy and Nonhydrogenic Rydberg Series in Monolayer Ws2. Phys. Rev. Lett. 2014, 113.

62. Berkelbach, T. C.; Reichman, D. R., Optical and Excitonic Properties of Atomically Thin Transition-Metal Dichalcogenides. In Annual Review of Condensed Matter Physics, Vol 9, Sachdev, S.; Marchetti, M. C., Eds. 2018; Vol. 9, pp 379-396.

63. Raja, A.; Chaves, A.; Yu, J.; Arefe, G.; Hill, H. M.; Rigosi, A. F.; Berkelbach, T. C.; Nagler, P.; Schuller, C.; Korn, T.; Nuckolls, C.; Hone, J.; Brus, L. E.; Heinz, T. F.; Reichman, D. R.;

Chernikov, A., Coulomb Engineering of the Bandgap and Excitons in Two-Dimensional Materials. Nature Commun. 2017, 8.

64. Stier, A. V.; Wilson, N. P.; Clark, G.; Xu, X. D.; Crooker, S. A., Probing the Influence of Dielectric Environment on Excitons in Monolayer Wse2: Insight from High Magnetic Fields. Nano Lett. 2016, 16, 7054-7060.

65. Klingshirn, C. F., Semiconductor Optics. Springer-Verlag Berlin Heidelberg: 2007.

66. Splendiani, A.; Sun, L.; Zhang, Y. B.; Li, T. S.; Kim, J.; Chim, C. Y.; Galli, G.; Wang, F., Emerging Photoluminescence in Monolayer Mos2. Nano Lett. 2010, 10, 1271-1275.

67. Hüser, F.; Olsen, T.; Thygesen, K. S., Quasiparticle Gw Calculations for Solids, Molecules, and Two-Dimensional Materials. Phys. Rev. B 2013, 87, 235132.

68. Onida, G.; Reining, L.; Rubio, A., Electronic Excitations: Density-Functional Versus ManyBody Green's-Function Approaches. Reviews of Modern Physics 2002, 74, 601-659.

69. Cho, Y.; Berkelbach, T. C., Environmentally Sensitive Theory of Electronic and Optical Transitions in Atomically Thin Semiconductors. Phys. Rev. B 2018, 97, 041409.

70. Thygesen, K. S., Calculating Excitons, Plasmons, and Quasiparticles in 2d Materials and Van Der Waals Heterostructures. 2D Mater. 2017, 4, 022004. 
71. Andersen, K.; Latini, S.; Thygesen, K. S., Dielectric Genome of Van Der Waals Heterostructures. Nano Lett. 2015, 15, 4616-4621.

72. Latini, S.; Winther, K. T.; Olsen, T.; Thygesen, K. S., Interlayer Excitons and Band Alignment in Mos2/Hbn/Wse2 Van Der Waals Heterostructures. Nano Lett. 2017, 17, 938-945.

73. Ugeda, M. M.; Bradley, A. J.; Shi, S.-F.; da Jornada, F. H.; Zhang, Y.; Qiu, D. Y.; Ruan, W.; Mo, S.-K.; Hussain, Z.; Shen, Z.-X.; Wang, F.; Louie, S. G.; Crommie, M. F., Giant Bandgap Renormalization and Excitonic Effects in a Monolayer Transition Metal Dichalcogenide Semiconductor. Nat. Mater. 2014, 13, 1091-1095.

74. Hüser, F.; Olsen, T.; Thygesen, K. S., How Dielectric Screening in Two-Dimensional Crystals Affects the Convergence of Excited-State Calculations: Monolayer Mos $\$\{\}_{-}\{2\} \$$. Phys. Rev. B 2013, 88, 245309.

75. Winther, K. T.; Thygesen, K. S., Band Structure Engineering in Van Der Waals Heterostructures Via Dielectric Screening: The G\$\{Updelta. 2017, 4, 025059.

76. Mortensen, J. J.; Hansen, L. B.; Jacobsen, K. W., Real-Space Grid Implementation of the Projector Augmented Wave Method. Phys. Rev. B 2005, 71, 035109.

77. Ryou, J.; Kim, Y.-S.; Kc, S.; Cho, K., Monolayer Mos $<$ Sub $>2</$ Sub $>$ Bandgap Modulation by Dielectric Environments and Tunable Bandgap Transistors. Sci. Rep. 2016, 6, 29184.

78. Griffin, A.; Harvey, A.; Cunningham, B.; Scullion, D.; Tian, T.; Shih, C.-J.; Gruening, M.; Donegan, J. F.; Santos, E. J. G.; Backes, C.; Coleman, J. N., Spectroscopic Size and Thickness Metrics for Liquid-Exfoliated H-Bn. Chem. Mater. 2018, 30, 1998-2005. 